\title{
MINIMAL SUBMANIFOLDS DEFINED BY FIRST-ORDER SYSTEMS OF PDE
}

\author{
J. M. LANDSBERG
}

\begin{abstract}
We study first-order PDE systems implying the second-order system for minimal submanifolds of a Euclidean $n$-space $\mathbb{R}^{n}$. We approach the problem geometrically by studying subsets $\Sigma$ of the Grassmannian which we call $m$-subsets, where we define $\Sigma$ to be an m-subset if all submanifolds of $\mathbf{R}^{n}$, whose Gauss map's image is contained in $\Sigma$, are automatically minimal. $\mathrm{m}$-subsets generalize the faces of calibrations studied by Harvey and Lawson. We also study linear first-order systems implying Laplace's equation, the infinitesimal version of the m-subset problem. Results include new examples of classes of minimal submanifolds admitting 'Weierstrass type' presentations in terms of holomorphic data; dimension restriction and rigidity theorems for m-subsets that extend to faces of calibrations; and showing certain codimension-two minimal submanifolds of $\mathbf{R}^{n}$ are stable using a nonconstant coefficient calibration argument.
\end{abstract}

\section{Introduction}

PDE. It was first observed by Riemann that one could obtain two solutions to Laplace's second-order equation

$$
u_{x x}+u_{y y}=0
$$

by solving the first-order Cauchy-Riemann system

$$
u_{x}=v_{y}, \quad u_{y}=-v_{x} \text {. }
$$

Since then, others have studied systems of second-order PDE having related first-order systems which imply the second-order system. By 'system A implies system B,' we mean solutions of A are automatically solutions of $B$.

A nonlinear example of this phenomena occurs in Yang-Mills theory in four dimensions. The vector bundles with self-dual connections (a firstorder system of PDE) satisfy the (second-order) Euler-Lagrange equations

Received June 8, 1990 and, in revised form, July 22, 1991.

Key words and phrases. Minimal submanifolds, calibrations, exterior differential systems. 
for the Yang-Mills functional. These vector bundles are not only critical points, but in fact are global minima of the functional.

We examine another case of a second-order PDE system which has firstorder systems implying it.

We study first-order systems whose solutions automatically solve the second-order minimal submanifold system. Some, but not all, of the classes of solutions which we get are global minima for the volume functional, in analogy with the situation in Yang-Mills. Along the way, we also study linear first-order systems implying Laplace's equation, as this is the linear version of the minimal submanifold system.

Geometry. Our problem may be rephrased geometrically as follows: Given $f: M^{n} \rightarrow \mathbf{R}^{n+s}$ any (oriented) submanifold, we have the Gauss map $\gamma_{f}: M \rightarrow G_{n, n+s}$ defined by $\gamma_{f}(x)=T_{f(x)} f(M)$. Here $G_{n, n+s}$ denotes the (oriented) Grassmannian of $n$-planes in $\mathbf{R}^{n+s}$.

The minimal submanifold system for an $n$-dimensional submanifold of $\mathbf{R}^{n+s}$ may be described geometrically as a restriction on the derivative of the Gauss map of the submanifold. Namely, $f$ is minimal if the mean curvature vector of $f$ is zero. The first-order systems we study may be described geometrically as restrictions on the image of the Gauss map.

The best known examples of first-order systems implying the minimal submanifold system are the equations for complex submanifolds. Considering $\mathbf{R}^{2(n+s)} \simeq \mathbf{C}^{n+s}$, a submanifold $M^{2 n}$ is complex if all its tangent planes are complex planes, i.e., if the image of its Gauss map is contained in the complex Grassmannian $G\left(\mathbf{C}^{n}, \mathbf{C}^{n+s}\right) \subset G_{2 n, 2(n+s)}$.

In fact, complex submanifolds are not only minimal, i.e., critical for the variation of volume, but are globally minimizing, i.e., actual minima for the volume functional. The proof that complex submanifolds are globally minimizing is due to Federer [7], and is based on Wirtinger's inequality. The proof uses Wirtinger's characterization of complex submanifolds; namely, they are the submanifolds of $\mathbf{C}^{n+s}$ on whose unit tangent planes the (appropriately normalized power of the) Kähler form assumes its maximum value.

Harvey and Lawson, in [8], noticed that the only properties of the Kähler form used in Federer's proof are that it is closed and unit-comass. (An $n$-covector $\phi$ is said to be unit comass if $\phi(E) \leq 1 \forall E \in G_{n, n+s}$, where we consider $E$ as a unit $n$-vector, and a differential form is unit-comass if it is pointwise unit-comass.) Harvey and Lawson call closed unit-comass forms calibrations. To each calibration $\phi$ there is a class of area minimizing submanifolds associated to it, namely those submanifolds on which 
$\phi$ restricts to be the volume form. (One says these submanifolds are calibrated by $\phi$.) Another way to describe the submanifolds calibrated by $\phi$ is to say their Gauss maps' images are contained in the subset of the Grassmann bundle on which $\phi$ assumes the value one. If $\phi$ is parallel, e.g., if our ambient space is $\mathbf{R}^{n+s}$ and $\phi$ is a constant coefficient differential form, then we may define the face of $\phi$ to be $\left\{E \in G_{n, n+s} \mid \phi(E)=1\right\}$ and submanifolds calibrated by $\phi$ are just those whose Gauss maps are contained in the face of $\phi$. Four new calibrations are studied in [8], and each has a large class of area minimizing submanifolds associated to it. (Few nonparallel calibrations have been studied; prior to this paper, their main use had been to prove certain cones in $\mathbf{R}^{n+s}$ are area minimizing, e.g., [10], [12].)

Definitions. We define $\Sigma \subset G_{n, n+s}$ to be an m-subset if $\gamma_{f(M)} \subset \Sigma$ implies that $f(M)$ is minimal (' $\mathrm{m}$ ' for minimal submanifold producing). In this case we will call $f(M)$ a $\Sigma$-manifold.

Overview. Part $\mathrm{I}$ is a study of the linear problem. Let $V$ and $W$ be vector spaces of dimensions $n$ and $s$ respectively, and let $V$ be equipped with an inner product. Let $C^{\infty}(V, W)$ denote the smooth $W$-valued functions on $V$. Consider the Laplacian as a second-order differential operator $\Delta: C^{\infty}(V, W) \rightarrow C^{\infty}(V, W)$. We would like to find linear first-order operators $D_{1}: C^{\infty}(V, W) \rightarrow C^{\infty}(V, \mathscr{R})$ such that $\operatorname{ker}\left(D_{1}\right) \subseteq \operatorname{ker}(\Delta)$, where $\mathscr{R}$ is some auxiliary vector space. We construct examples of such operators $D_{1}$ and prove some general lemmas restricting their existence. For example, any such $D_{1}$ must correspond to at least $\max (n, s)$ equations (Lemmas I.2.3 and I.2.4). Moreover, when the number of equations is exactly $\max (n, s)$, then, if $s \geq n, D_{1}$ is the Dirac operator of $\mathrm{Cl}^{\text {even }}(V)$ on $W$, and, if $n \geq s, V$ is the dimension of a $\mathrm{Cl}^{\text {even }}(W)$ module (Lemma I.2.5). We also show that if $n$ or $s=2$, then the system must correspond to the Cauchy-Riemann equations (assuming the tableau is involutive) and classify cases when $n=3$ and $s$ is small (Theorems I.3.1 and I.3.2).

In Part II, we go from the infinitesimal solutions of Part I to local solutions. That is, we take some of the known linear first-order systems implying the Laplace system and look for corresponding first-order systems implying the minimal submanifold system. We explain how these systems correspond geometrically to the submanifolds of the Grassmannian which we call $\mathrm{m}$-subsets. The results are striking:

We show that in (real) dimension and codimension two, there are many (arbitrary functions worth of) m-subsets having the same linearized firstorder system as the complex Grassmannians. Homogeneous examples of 
these m-subsets in codimension two are given by Theorem II.3.10. The resulting $\Sigma$-manifolds have an interesting description. They may be obtained by a 'twistor'-type construction; one transports holomorphic data on an auxiliary space to Euclidean space.

In contrast, for all the other standard linear systems, there are essentially unique m-subsets corresponding to the standard faces (Theorems II.3.1 and II.3.1*). Moreover, we show that all other linear systems solving the infinitesimal problem that have $\max (n, s)$ equations (which are the fewest equations possible, as shown in Part I) have no corresponding m-subsets (where we are looking for m-subsets in $G_{n, n+s}$ ). This puts further restrictions on the potential dimensions of faces.

Finally in the appendix we construct (local) calibrations for a certain class of $\Sigma$-manifolds. Namely, let $\Sigma$ be a degenerate $\mathrm{SO}(3)$ orbit of an $(m-2)$-plane in $G_{m-2, m}$, where $\mathrm{SO}(3)$ acts irreducibly on $\mathbf{R}^{m}$. These $\Sigma$-manifolds are studied in detail in [9].

For each such $\Sigma$-manifold, we construct a nonconstant coefficient (local) calibration tailor-made to calibrate that solution. In particular, this shows that these $\Sigma$-manifolds are stable.

The results of this paper and [9] are based on the author's doctoral dissertation under the direction of Robert Bryant. It is a pleasure to thank him for support and inspiration, and in particular for suggesting that faces of calibrations might be generalized.

\section{PART I: LINEAR STUDY}

In this part we address the question:

(*) What linear first-order systems of PDE for $s$ functions of $n$ variables have the property that their solutions are automatically solutions of the Laplace system for $s$ functions of $n$ variables?

Example 1. We already have seen that the Laplace system for two functions of two variables is implied by the Cauchy-Riemann equations.

Example 2. The Laplace system for three functions, say $u, v, w$, of three variables, say $x, y, z$, is implied by

$$
u_{x}+v_{y}+w_{z}=0, \quad u_{y}=v_{x}, \quad u_{z}=w_{x}, \quad v_{z}=w_{y} .
$$

This is the linear system corresponding to the special Lagrangian system in $\mathbf{R}^{6}$, first studied in [8]. (See Part II, $\S 1$ for the definition of the special Lagrangian system.) 
We rephrase $(*)$ more precisely: Let $V$ and $W$ be vector spaces. Throughout this part, $V$ will always be of dimension $n, W$ of dimension $s$, and $V$ will come equipped with an inner product. Let $C^{\infty}(V, W)$ denote the smooth $W$-valued functions on $V$. Consider the Laplacian as a second-order differential operator, $\Delta: C^{\infty}(V, W) \rightarrow C^{\infty}(V, W)$. We would like to find first-order operators $D_{1}: C^{\infty}(V, W) \rightarrow C^{\infty}(V, \mathscr{R})$, such that $\operatorname{ker}\left(D_{1}\right) \subseteq \operatorname{ker}(\Delta)$, where $\mathscr{R}$ is some auxiliary vector space.

Example 3. Let $W$ be a $\mathrm{Cl}^{\text {even }}(V)$ module, $\mathscr{R}=W$, and $D_{1}=$ $\sum_{j} e_{j} \partial_{j}$ the Dirac operator (see [15]).

In Part II we will see that $(*)$ is the infinitesimal version of the following question: What subsets of the Grassmannian $G_{n, n+s}$ guarantee minimality for any $n$-dimensional submanifold of $\mathbf{R}^{n+s}$ whose Gauss map's image lies in it?

In $\S 1$ we define the problem in the language of exterior differential systems and discuss several examples; in $\S 2$ we prove some general results which place restrictions on the dimensions of such first-order systems; and finally in $\S 3$, the systems, which we will call $m$-tableaux, are classified in some special cases.

\section{EDS point of view and examples}

It will be useful to rephrase our question using language taken from exterior differential systems, henceforth called EDS. This will enable us to take advantage of the EDS machinery directly, and, more importantly, the EDS point of view will enable us to rephrase the problem geometrically, as the first-order systems expressed as m-tableaux have a natural identification with candidate tangent spaces to m-subsets as we will see in Part II. (Here and elsewhere in this paper, the word 'candidate' applied to an object should be taken to mean the object satisfies some necessary condition.)

We think of the equations as placing relations on derivatives, and look at the dual object, the space of admissible derivatives.

In the language of EDS, a linear subspace $A \subset \operatorname{Hom}(V, W)$ is called a tableau. It specifies a linear first-order homogeneous system of PDE for maps $V \rightarrow W$, whose solutions are those maps whose Jacobian lies in $A$ at each point. To get a set of equations giving this system, simply take a basis of $A^{\perp}$ and identify $\left(A^{\perp}\right)_{j}^{i}$ with the partial of the $i$ th function with respect to the $j$ th variable.

Example $1^{\prime}$. The Cauchy-Riemann system $u_{x}=v_{y}, u_{y}=-v_{x}$ has tableau 


$$
A=\left\{\left(\begin{array}{cc}
a & -b \\
b & a
\end{array}\right) \mid a, b \in \mathbf{R}\right\} .
$$

Here, solutions are $\left(\begin{array}{l}u(x, y) \\ v(x, y)\end{array}\right)$ such that $\left(\begin{array}{l}d u \\ d v\end{array}\right) \in A\left(\begin{array}{l}d x \\ d y\end{array}\right) \forall x, y \in \mathbf{R}$.

How does one relate the notions of systems given by tableaux to systems given by operators $D_{1}$ ? Given a tableau $A$, we produce an operator $D_{1}$ as follows: Functions $f: V \rightarrow W$ in the kernel of $D_{1}$ should satisfy the defining relations of $A$. Take $\mathscr{R}$ to be the space of defining relations of $A$, i.e., $\mathscr{R}=\left(W \otimes V^{*}\right) / A$, and let $D_{1}$ be the map $f \mapsto[\operatorname{Jac}(f)]$. Then $f: V \rightarrow W$ will be in the kernel of $D_{1}$ if and only if $f$ is a solution to the differential system specified by $A$.

A tableau of order $2, \tilde{A} \subset W \otimes \operatorname{Sym}^{2} V^{*}$, specifies a second-order linear homogeneous system of PDE for maps $V \rightarrow W$; namely, those maps whose second-order partials lie in $\widetilde{A}$ at each point.

In particular, the Laplace system for $W$-valued functions on $V$ is given by

$$
\widetilde{A}=\left\{P \in W \otimes \operatorname{Sym}^{2} V^{*} \mid \operatorname{trace}(P)=0\right\}=W \otimes \operatorname{Sym}_{0}^{2} V^{*},
$$

where trace: $W \otimes \operatorname{Sym}^{2} V^{*} \rightarrow W$ is with respect to the induced metric on $V^{*}$.

Given a tableau $A$, the space of admissible second order terms in a power series solution to the system which $A$ specifies is called the prolongation of $A$. We denote it by $A^{(1)}$, i.e.,

$$
\begin{aligned}
A^{(1)} & =\left\{P \in W \otimes \operatorname{Sym}^{2} V^{*} \mid \frac{\partial P}{\partial x} \in A \forall x \in V^{*}\right\} \\
& =\left(A \otimes V^{*}\right) \cap\left(W \otimes \operatorname{Sym}^{2} V^{*}\right) .
\end{aligned}
$$

Notice that $A^{(1)}$ is a tableau of order two, and thus specifies a secondorder system. We want to find the first-order systems whose prolonged system includes the Laplace system. Thus in the language of EDS our basic question becomes: What are the tableaux $A$ such that $A^{(1)}$ is traceless?

Definition. We say $A \subset W \otimes V^{*}$ is an m-tableau if $A^{(1)}$ is traceless.

Example 2'. $A \subset \operatorname{Hom}(V, V)$ such that $\operatorname{tr}(A)=0$ and ${ }^{t} A=A$ gives the $\mathrm{m}$-tableau corresponding to the special Lagrangian system. (If $n=\operatorname{dim}(V)=3$, we recover Example 2.)

Example $3^{\prime}$. The associated m-tableaux to the Dirac operators of Example 3 are described explicitly in $\S 2$ by (2.1). Proposition 2.7 gives the identification $\mathscr{R}=W$. Among these are the Cauchy-Riemann m-tableaux (Example $1^{\prime}$ ), the associative $m$-tableau, the coassociative $\mathrm{m}$-tableau, and 
the Cayley m-tableau (respectively corresponding to the linearized equations for associative, coassociative, and Cayley submanifolds; see Part II, $\S 1)$.

Example 4. Harmonic forms that are both $d$ and $d^{*}$ closed. Let $W=$ $\Lambda^{k} V, \mathscr{R}=\Lambda^{k+1} V \oplus \Lambda^{k-1} V$, and

$$
D_{1}=d \oplus d^{*}: \Omega^{k}(V) \rightarrow \Omega^{k+1}(V) \oplus \Omega^{k-1}(V),
$$

where $\Omega^{k} V=C^{\infty}\left(V, \Lambda^{k} V\right)$. To identify $\mathscr{R}$ with $\left(W \otimes V^{*}\right) / A$ for some m-tableau $A$, consider the map

$$
\begin{gathered}
V^{*} \otimes \Lambda^{k} V \rightarrow \Lambda^{k+1} V \oplus \Lambda^{k-1} V, \\
\left.(v, \alpha) \mapsto v^{\sharp} \wedge \alpha \oplus v\right\lrcorner \alpha,
\end{gathered}
$$

where $v^{\sharp}$ is the metric dual to $v$ and $\lrcorner$ denotes interior multiplication. Now let $A$ be the kernel of this map.

The case $k=1, V^{*} \otimes \Lambda^{1} V \rightarrow \Lambda^{2} V \oplus \mathbf{R}$ gives the special Lagrangian m-tableau (Example $2^{\prime}$ ), as the kernel in this case (choosing orthonormal bases) is $A$ such that ${ }^{t} A=A$ because $A$ must be in the kernel of $d$ (i.e., $v^{\sharp} \wedge \alpha=0$ ), and $\operatorname{tr}(A)=0$ because $A$ must be in the kernel of $d^{*}$ (i.e., $v\lrcorner \alpha=0$ ). So we may think of the equations for special Lagrangian submanifolds as a nonlinear version of the equations for $d$ and $d^{*}$ closed one-forms.

In the case $k=2$, the first new case would be $n=4$, but in fact this turns out to produce an m-tableau that is two copies of the coassociative tableau because $\Lambda^{2} \mathbf{R}^{4}$ splits orthogonally into self- and anti-self-dual components. For $n>4$ we get new examples of m-tableaux. To determine whether there are m-subsets associated to them is a difficult problem. The prospects are not good for finding m-subsets because, in general, for dimension reasons, there are no homogeneous m-subsets associated to these m-tableaux.

Remark. This example illustrates the contrast between the compact and noncompact situations. If a manifold is compact then the harmonic forms are automatically $d$ and $d^{*}$ closed. It may be interesting to study what first-order systems implying second-order systems become automatic in some compact setting and vice-versa.

The m-tableaux of the examples above are all involutive in the sense of EDS. Roughly, a tableau is involutive if there are no 'hidden' equations that arise by differentiating the given equations; the space of solutions to the system is as large as one could näively hope it to be. Involutivity is a generalization of integrability, guaranteeing not just that there is a solution, 
but using the Cartan-Kähler theorem, one can describe the moduli space of solutions. The involutive $m$-tableaux provide a natural class of $m$-tableaux to study and in what follows we will focus on them.

\section{Observations about m-tableaux}

The characteristic variety of an m-tableau. A useful condition for a tableau to be an m-tableau is given by its characteristic variety. In general the characteristic variety of a differential system is a generalization of the set of characteristic directions of a linear system of PDE. It is the set of hyperplanes in a given integral element whose enlargement to an integral element is not unique. We review from [4]:

Given a tableau $A \subset W \otimes V^{*}$, let $V^{\mathrm{C}}$ denote the complexification of $V$, etc. and define

$$
\Xi_{A}:=\left\{[\xi] \in \mathbf{P}\left(V^{\mathbf{C} *}\right) \mid \exists w \in W^{\mathbf{C}}, w \neq 0, \text { such that } w \otimes \xi \in A^{\mathbf{C}}\right\} .
$$

(In [4] this is referred to as the complex characteristic variety, but since it is the only one we will be using, we drop the adjective.)

Given $\xi \in V^{\mathbf{C} *}$, to determine if $[\xi] \in \Xi_{A}$ we need to know if there exists a $w \in W^{\mathbf{C}}$ such that $w \otimes \xi \in A^{\mathbf{C}}$.

Choose a basis $b^{1}, \cdots, b^{r}$ of $\left(A^{\mathrm{C}}\right)^{\perp}$ (where $r$ is the codimension of $A$ in $\left.W \otimes V^{*}\right)$. Now $w \otimes \xi \in A^{\mathbf{C}}$ if and only if $b^{a}(w \otimes \xi)=0,1 \leq a \leq r$, i.e., iff $w \otimes \xi \in \operatorname{ker}(\sigma)$, where

$$
\begin{aligned}
\sigma: W^{\mathbf{C}} \times V^{\mathbf{C} *} \rightarrow \mathbf{C}^{r}, \\
(w, \xi) \mapsto b(w \otimes \xi)=\left(\begin{array}{c}
b^{1}(w \otimes \xi) \\
\vdots \\
b^{r}(w \otimes \xi)
\end{array}\right) .
\end{aligned}
$$

(This is just the map $W^{\mathbf{C}} \times V^{\mathbf{C} *} \rightarrow W^{\mathbf{C}} \otimes V^{\mathbf{C} *} / A^{\mathbf{C}}$ in coordinates.) Now $[\xi] \in \mathbf{\Xi}_{A}$ if there exists some $w \in W^{\mathbf{C}}$ such that $(w, \xi) \in \operatorname{ker}(\sigma)$. Let $\sigma_{\xi}$ be the map

$$
\begin{aligned}
\sigma_{\xi}: W^{\mathbf{C}} \rightarrow \mathbf{C}^{r}, \\
w \mapsto b(w \otimes \xi) .
\end{aligned}
$$

We see that $[\xi] \in \Xi_{A}$ iff $\sigma_{\xi}$ has a kernel, i.e., iff $\operatorname{rank}\left(\sigma_{\xi}\right)<s$, i.e., iff the $s \times s$ minors of $\sigma_{\xi}$ are zero.

This shows that (the deprojectivization of) $\Xi_{A}$ is a determinental variety whose defining equations have real coefficients and gives an effective way of computing it. 
The characteristic variety of the Laplace system for $s$ functions of $n$ variables is the null quadric $\left(\langle\right.$,$\rangle denotes the bilinear form on V^{\mathrm{C} *}$ induced from the metric on $V^{*}$ ):

$$
Q=\left\{[\xi] \in \mathbf{P}\left(V^{\mathbf{C} *}\right) \mid\langle\xi, \xi\rangle=0\right\} .
$$

This cannot be directly seen by the definition above which is only valid for (first-order) tableaux, but any reasonable definition gives $Q$ (e.g., see the general definition of the characteristic variety of an EDS in Chapter 5 of [4]).

For systems without integrability conditions, such as the ones here, the characteristic variety does not change under prolongation, i.e., $\Xi_{A^{(1)}}=\Xi_{A}$. Thus: $A$ tableau is an $m$-tableau if and only if its characteristic variety is contained in the null quadric $Q$.

General lemmas. When we actually try to solve for m-subsets we will only be concerned with m-tableaux up to $\mathrm{Gl}(W) \times \mathrm{SO}(V)$ equivalence, so in what follows we will consider two $m$-tableaux equivalent if they differ by an $\mathrm{Gl}(W) \times \mathrm{SO}(V)$ action. (Normally when studying differential systems, one would consider tableaux equivalent if they differ by a $\mathrm{GL}(W) \times \mathrm{GL}(V)$ action, but we only consider linear transformations of $V$ preserving the metric and thus the trace.)

Assume we have chosen an $A$-generic basis for $V^{*}$ (see [4] for the definition of $A$-generic), say $\left\{x_{1}, \cdots, x_{n}\right\}$. Let $V_{k}^{*}:=\operatorname{span}\left\{x_{k+1}, \cdots, x_{n}\right\}$ and $A_{k}:=A \cap\left(W \otimes V_{k}^{*}\right)$.

Lemma 2.1. If $A$ is an m-tableau, then $A_{k}$ is too, considered both as a tableau in $W \otimes V^{*}$ and as a tableau in $W \otimes V_{k}^{*}$.

Proof.

$A_{k}^{(1)}=\left\{P \in W \otimes S^{2} V_{k}^{*} \mid \partial P / \partial x^{i} \in A, \partial^{2} P / \partial x^{i} \partial x^{j}=0, k+1 \leq i, j \leq n\right\}$

which is a linear subspace of $A^{(1)} \subset W \otimes S^{2} V^{*}$ and is thus in the kernel of the linear map trace $W \otimes S^{2} V^{*} \rightarrow W$ because $A^{(1)}$ is; the same holds restricted to $W \otimes \operatorname{Sym}^{2} V_{k}^{*}$.

Corollary 2.2. If $A$ is an involutive m-tableau, then $A_{k}$ is too.

Proof. This is just because if $A$ is involutive then $A_{k}$ is too (see [4]).

The characters $s_{k}, 1 \leq k \leq n$, of a tableau $A$ are defined inductively by

$$
s_{1}+\cdots+s_{k}=\operatorname{dim} A-\operatorname{dim} A_{k} .
$$

For any tableau $A$ one has

$$
\operatorname{dim} A^{(1)} \leq s_{1}+2 s_{2}+\cdots+n s_{n}
$$


and $A$ is involutive if equality holds. If $s_{b}$ is the last nonzero character of an involutive tableau $A$, then, roughly speaking, solutions of the differential system depend on $s_{b}$ arbitrary functions of $b$ variables. The following lemma says that solutions to differential systems given by m-tableaux can depend on at most $n-1$ variables, and we will see that these extreme cases are rare, essentially all given by equation (2.1) below.

Lemma 2.3 (R. Bryant). An m-tableau must have $s_{n}=0$ and thus be at least of codimension $s$ in $W \otimes V^{*}$.

Proof. Version 1: $A_{n-1}$ must be an m-tableau also but $A_{n-1}^{(1)}=\{w \otimes$ $\left.x_{n} \circ x_{n} \mid w \otimes x_{n} \in A_{n-1}\right\}$, so $A_{n-1}=0$ for any m-tableau. Version 2: $s_{n} \neq 0 \Rightarrow \Xi_{A}=\mathbf{P}\left(V^{\mathbf{C} *}\right)$.

The dual tableau. For the moment, forget that $V$ has a metric on it. Given a linear subspace $A \subset W \otimes V^{*}$, we have seen that we may consider it as a tableau in $\operatorname{Hom}(V, W)$, giving a PDE system for maps $V \rightarrow W$. We may also consider it as a tableau in $\operatorname{Hom}\left(W^{*}, V^{*}\right)$, giving a $\operatorname{PDE}$ system for maps $W^{*} \rightarrow V^{*}$. When we think of $A$ in the second way we will call it the dual tableau and denote it $A^{*}$. Notice that

$$
\Xi_{A^{*}}=\left\{[w] \in \mathbf{P} W^{\mathbf{C}} \mid \exists \xi \in V^{\mathbf{C} *}, \xi \neq 0, \text { such that } w \otimes \xi \in A^{\mathbf{C} *}\right\} .
$$

In particular, we have

$$
\Xi_{A^{*}}^{\mathbf{R}}=\varnothing \Leftrightarrow \Xi_{A}^{\mathbf{R}}=\varnothing,
$$

where $\Xi_{A}^{\mathbf{R}}$ denotes the real points of $\Xi_{A}$.

More general lemmas.

Lemma 2.4. If $A$ is an $m$-tableau, then $\operatorname{codim}(A) \geq \max (n, s)$.

Proof. We have already seen the case $s \geq n$. If $n>s$, say $\operatorname{codim}(A)$ $<n$, then $A^{*}$ must have $s_{s} \neq 0 \Rightarrow \Xi_{A^{*}}^{\mathbf{R}} \neq \varnothing \Rightarrow \Xi_{A}^{\mathbf{R}} \neq \varnothing \Rightarrow A$ is not an m-tableau.

In fact we conjecture the stronger statement: $\Xi_{A}$ is contained in a quadric with no real points iff $\Xi_{A^{*}}$ is too. This would imply that: $A$ is an $m$-tableau with respect to some metric on $V$ iff $A^{*}$ is an $m$-tableau with respect to some metric on $W^{*}$.

Notice that the necessity of ellipticity (i.e., $\Xi_{A}^{\mathbf{R}}=\varnothing$ ) implies that if $A$ is an m-tableau of maximal dimension, then, when $\operatorname{codim}(A)=n, A^{*}$ must be involutive, and, when $\operatorname{codim}(A)=s, A$ must be involutive.

Lemma 2.5. Let $A$ be an m-tableau.

(i) (R. Bryant) If $s \geq n$ and $\operatorname{codim}(A)=s$, then $s$ is the dimension of a $\mathrm{Cl}_{n-1}$ module.

(ii) If $n \geq s$ and $\operatorname{codim}(A)=n$, then $n$ is the dimension of a $\mathrm{Cl}_{s-1}$ module. $\left(\mathrm{Cl}_{m}\right.$ denotes the Clifford algebra over $\mathbf{R}^{m}$.) 
The proof will follow from the following general EDS lemma:

Lemma 2.6. Let $A$ be an elliptic tableau of codimension $s$ in $W^{s} \otimes$ $V^{n *}$. Then $s$ must be the dimension of a $\mathrm{Cl}_{n-1}$ module.

Proof. Ellipticity implies $s_{n}=0$. There exist a basis $\pi_{1}, \cdots, \pi_{n}$ of $V^{*}$ and endomorphisms $C_{1}, \cdots, C_{n-1}$ of $W$ such that $A$ is the kernel of the linear mapping

$$
\begin{gathered}
\sigma: W \otimes V^{*} \rightarrow W, \\
\sigma\left(w^{j} \otimes \pi_{j}\right)=w_{n}-C_{j}\left(w^{j}\right)
\end{gathered}
$$

with $w^{1}, \cdots, w^{n} \in W$. We see that $A$ is elliptic if and only if $w, C_{1} w$, $\cdots, C_{n-1} w$ are linearly independent vectors for all nonzero $w \in W$ or, equivalently, for all $W$ belonging to the unit sphere $S^{s-1}$ in $W$ (with respect to some fixed metric on $W$ ). For $w \in S^{s-1}$, let $C_{j}^{\prime} w=$ $C_{j} w-\left\langle C_{j} w, w\right\rangle w$ be the orthogonal projection of $C_{j} w$ onto the hyperplane orthogonal to $w$. The vectors $w, C_{1} w, \cdots, C_{n-1} w$ are linearly independent for $w \in S^{s-1}$ if and only if $C_{1}^{\prime} w, \cdots, C_{n-1}^{\prime} w$ are linearly independent. Thus if $A$ is elliptic, the maps $w \mapsto C_{j}^{\prime} w$ for $w \in S^{s-1}$ give us $n-1$ independent vector fields on $S^{s-1}$; the value of the $j$ th vector field at $w \in S^{s-1}$ is $C_{j}^{\prime} w$.

Now we appeal to a result of Adams [1] which says that there exist $m$ linearly independent vector fields on $S^{s-1}$ only if $\mathrm{Cl}_{m}$ has a representation of dimension $s$. q.e.d.

Lemma 2.5 now follows by applying Lemma 2.6 to $A$ and $A^{*}$. q.e.d. To explicitly see examples of these extreme m-tableaux, write

$$
A=\left(\pi_{1}, \cdots, \quad \pi_{n-1}, \quad J_{1} \pi_{1}+\cdots+J_{n-1} \pi_{n-1}\right),
$$

where

$$
\pi_{k}=\left(\begin{array}{c}
\pi_{k}^{1} \\
\vdots \\
\pi_{k}^{s}
\end{array}\right)
$$

and we have chosen bases $\left\{e^{i}\right\}$ of $V$ and $\left\{f_{a}\right\}$ of $W$ such that $\pi_{j}^{a}$ is the coefficient of $e^{i} \otimes f_{a}$, and $J_{q} \in M_{s \times s}(\mathbf{R})(\cong \operatorname{End}(W))$ is such that $J_{q}^{2}=-$ Id and $J_{q} J_{r}+J_{r} J_{q}=0, r \neq q . A$ is an involutive m-tableau and, with an appropriate metric on $W$, so is $A^{*}$. We will call such m-tableaux Dirac m-tableaux, and their duals Dirac ${ }^{*} m$-tableaux.

Corollary 2.7. If $A$ is an involutive $m$-tableau whose characters are such that $\kappa=s_{p}=s_{p+1}=\cdots=s_{n-1}$, where $\kappa$ is the character of $A$, then $\mathbf{R}^{\kappa}$ must be a $\mathrm{Cl}_{n-1-p}$ module. 
Proof. This is because $A_{p-1}$ is a Dirac m-tableau.

Proposition 2.8. The Clifford module structure of a Dirac m-tableau is canonical.

Proof. For $x, y \in V$ let $m(x y): W \rightarrow W$ be defined by

$$
W \stackrel{\left.|y|^{2}(y\lrcorner\right)^{-1}}{\longrightarrow} A^{\perp} \stackrel{(x\lrcorner)}{\longrightarrow} W
$$

where $A^{\perp} \subset W^{*} \otimes V$ and $\left.x\right\lrcorner(w \otimes v)=x(v) w$.

One now just checks that $m(x y)+m(y x)=2\langle x, y\rangle$ Id in a basis by using the basis for $A$ of (2.1). The key to the computation is that ${ }^{t} J_{i}=-J_{i}$. For a more general result and abstract proof, see [6]. q.e.d.

Examples of $m$-tableaux of this type are Cauchy-Riemann m-tableaux when $\operatorname{dim}(V)=2$, and the linearized equations for associative submanifolds in $G_{3,7}$ and Cayley submanifolds in $G_{4,8}$. In general, they are the tableaux of the Dirac operators discussed in Example $3^{\prime}$ of $\S 1$.

Lemma 2.9. The character $\kappa$ of an involutive $m$-tableau must be even.

We give two proofs.

Proof 1. By Lemma 2.1 it is sufficient to verify (2.9) under the assumption that $s_{1}=s, s_{2}=0$. Write

$$
A=\left(\pi, C_{2} \pi, \cdots, C_{n} \pi\right)=\left(\pi_{i}^{a}\right), \quad \text { where } \pi=\left(\begin{array}{c}
\pi^{1} \\
\vdots \\
\pi^{s}
\end{array}\right)
$$

(again we have chosen bases $e^{i}$ of $V$ and $f_{a}$ of $W$ such that $\pi^{a}$ is the coefficient of $\left.e^{1} \otimes f_{a}\right)$, and the $C_{k} \in M_{s \times s}(\mathbf{R})(\cong \operatorname{End}(W))$ are fixed endomorphisms determined by $A$. Write

$$
P_{j k}=\left(\begin{array}{c}
p_{j k}^{1} \\
\vdots \\
p_{j k}^{s}
\end{array}\right)
$$

for the coefficients of an integral element, i.e., an element of $A^{(1)}$. Since integral elements are in $W \otimes \operatorname{Sym}^{2} V^{*}$, we have $P_{i j}=P_{j i}$, and since integral elements are in $A \otimes V^{*}$, we have

$$
P_{i j}=C_{i} C_{j} P_{11}
$$

Furthermore,

$$
\operatorname{trace}\left(A^{(1)}\right)=\left(\mathrm{Id}+C_{2}^{2}+\cdots+C_{n}^{2}\right) P_{11} .
$$

$P_{i j}=P_{j i}$ requires that all the $C_{i}$ commute, i.e., that they share simultaneous eigenspaces. If $s$ is odd they must have a real simultaneous eigenspace 
with real eigenvalues, and thus their squares must have a simultaneous eigenspace with positive eigenvalues. However for $\operatorname{trace}\left(A^{(1)}\right)$ to be zero, there must be negative eigenvalues in the sum of the squares to cancel the +1 eigenvalue coming from Id, thus $s$ cannot be odd.

Proof 2 (R. Bryant). Recall from [4] that for involutive systems, the degree of the characteristic variety is the character of the system. We will show that for any involutive m-tableau $A$, the degree of $\Xi_{A}$ is even. Recall from algebraic geometry that the degree of a subvariety of projective space is the number of points of intersection with a generic plane of complementary dimension (counted with multiplicity).

Furthermore $\operatorname{deg}(X \cap Y)=\operatorname{deg}(X) \operatorname{deg}(Y)$ for transverse intersections. Intersect $\Xi_{A}$ with transverse real hyperplanes to reduce to the case of $\Xi_{A} \subset Q_{2}=\left\{\xi_{1}^{2}+\xi_{2}^{2}+\xi_{3}^{2}=0\right\}$.

Now $\Xi_{A}$ will be $d=\operatorname{deg}\left(\Xi_{A}\right)$ points. The defining equations for $\Xi_{A}$ are real, so if $\left[\xi_{1}, \xi_{2}, \xi_{3}\right]$ is in $\Xi_{A}$, then so is $\left[\bar{\xi}_{1}, \bar{\xi}_{2}, \bar{\xi}_{3}\right]$, and since there are no real points on $Q$, the points come in pairs.

\section{Classification in low dimensions}

The problem of classifying m-tableaux (or even involutive m-tableaux) naively involves solving $\left(\begin{array}{l}n \\ 2\end{array}\right)$ polynomial equations of degree $n-1$ in matrices of dimensions $s \times s$.

Lemma 2.1 simplifies this task substantially by allowing us to work inductively. If one has classified the case $\operatorname{dim} V=n, \operatorname{dim} W=s$ then the case $\operatorname{dim} V=n+1$ will involve $n-1$ quadratic equations of $s \times s$ matrices. The caveat is that one must solve such a set for each solution in the $(n, s)$ case.

Since we are restricting to involutive $m$-tableaux, things are made a little simpler by the use of Guillemin normal form (a preferred type of basis for a tableau, see [4, p. 141]), and we may work case by case according to the characters and the ranks of various matrices.

Although no one case is very difficult, the plethora of cases presents a problem. Another difficulty encountered is that new m-tableaux may be built out of old ones, and what we are really interested in is classifying 'irreducible' m-tableaux. For example, although no new m-tableaux occur when $n=3$ and $s_{2}=2$, there are many solutions to the equations obtained by 'stacking' Cauchy-Riemann m-tableaux and special Lagrangian $\mathrm{m}$-tableaux. Although it is just an annoyance from the point of view of the linear problem, 'stacking' m-tableaux can occasionally lead to interesting 
solutions of the nonlinear (i.e., m-subset) problem, e.g., the examples in $\S 5$ of Part II.

There are no m-tableaux for $n=1$ (e.g., by Lemma 8). Similarly there are none when $s=1$. Although obvious, these cases illustrate a point about how the general method of studying first-order systems that imply second-order systems works, namely we are taking independent copies of Laplace's equation and looking at a class of solutions that are somehow 'tied' together. Since the character of an involutive m-tableau must be even, we see by Lemma 4 that any involutive m-tableau in the case $n=2$ must be of the form $(\pi J \pi), J^{2}=-I d$, i.e., a Cauchy-Riemann mtableau.

In general it is easier to proceed by fixing $n$ and allowing $s$ to vary, but it is worth noting that Lemma 7 allows us to determine the case $s=2$. In fact, involutive m-tableaux with $s=2$ must have characters $s_{1}=\cdots=$ $s_{p}=2, s_{p+1}=\cdots=s_{n}=0$. Moreover it is easy to show any such m-tableau must be a Cauchy-Riemann m-tableau.

The rest of this part is devoted to the case of involutive m-tableaux for $n=3$, although this has implications for larger $n$ (e.g., Theorem 3.3). To produce a substantial further classification it would probably be better to try a different approach, perhaps using the algebraic geometry of the characteristic variety (although using the characteristic variety naively is even worse in the sense that it involves more equations of higher degree).

Theorem 3.1. The only involutive $m$-tableaux that occur in $G_{3, s+3}$ having $s_{2}=2$ are reducible to products of a special Lagrangian $m$-tableau and Cauchy-Riemann m-tableaux and these can only occur when $s$ is odd and $\geq 3$.

Theorem 3.2. The only involutive $m$-tableaux that occur in $G_{s, s+3}$ having character $\kappa=s_{2}=s-1$ are products of a special Lagrangian m-tableau and $\mathbf{H}$-Dirac m-tableaux. In particular, $s \equiv 3 \bmod 4$.

Theorem 3.3. The following are maximal dimensions for involutive $m$ tableaux:

$\begin{array}{cccccccc}s \backslash n & 2 & 3 & 4 & 5 & 6 & 7 & 8 \\ & & & & & & & \\ 2 & 2 & 2 & 4 & 4 & 6 & 6 & 8 \\ 3 & 2 & 5 & 8 & 8 & 10 & 13 & 16 \\ 4 & 4 & 8 & 12 & 12 & 16 & & \\ 5 & 4 & 8 & 12 & 14 & & & \\ 6 & 6 & 10 & 16 & & & & \\ 7 & 6 & 13 & & & & & \\ 8 & 8 & 16 & & & & & \end{array}$


Remark. For most cases in the chart, these are the largest possible dimensions of all $\mathrm{m}$-tableaux because any $\mathrm{m}$-tableau of such dimension or larger must be involutive.

We now set up the notation to enable us to prove the theorems. If $A$ has characters $s_{1}=s, s_{2}=\kappa, s_{3}=0$, we may choose bases $e^{i}$ of $V$ and $f_{a}$ of $W$ such that we may write

$$
A=\left(C_{1} \Pi_{1}+E_{1} \Pi_{2}, C_{2} \Pi_{1}+E_{2} \Pi_{2}, C_{3} \Pi_{1}+E_{3} \Pi_{2}\right),
$$

where

$$
\Pi_{1}=\left(\begin{array}{c}
\pi_{1}^{1} \\
\vdots \\
\vdots \\
\vdots \\
\pi_{1}^{s}
\end{array}\right), \quad \Pi_{2}=\left(\begin{array}{c}
\pi_{2}^{1} \\
\vdots \\
\pi_{2}^{\kappa} \\
0 \\
\vdots \\
0
\end{array}\right), \quad C_{i}, E_{i} \in M_{s \times s}(\mathbf{R})
$$

( $\pi_{i}^{a}$ is the coefficient of $e^{i} \otimes f_{a}$ ), $\pi_{1}^{1}, \cdots, \pi_{1}^{s}, \pi_{2}^{1}, \cdots, \pi_{2}^{\kappa}$ may be freely specified, and $C_{a}, E_{a}$ are fixed endomorphisms determined by $A$.

Let

$$
P_{i j}=\left(\begin{array}{c}
p_{i j}^{1} \\
\vdots \\
p_{i j}^{s}
\end{array}\right),
$$

where the $p_{i j}^{a}$ denote coordinates on $W \otimes \operatorname{Sym}^{2} V^{*}$.

On an integral element $P_{11},\left(E_{2} P_{12}\right)$, and $\left(E_{2} P_{22}\right)$ may be freely specified. The other $P_{i j}$ are determined by

$$
\begin{aligned}
P_{i j} & =C_{i} P_{1 j}+E_{i} P_{2 j} \\
& =C_{i}\left(C_{j} P_{11}+E_{j} P_{12}\right)+E_{i}\left(C_{j} P_{21}+E_{j} P_{22}\right)+E_{i} C_{j}\left(C_{2} P_{11}+E_{2} P_{12}\right) \\
& =\left(C_{i} C_{j}+E_{i} C_{j} C_{2}\right) P_{11}+\left(C_{i} E_{j}+E_{i} C_{j} E_{2}\right) P_{12}+\left(E_{i} E_{j}\right) P_{22} .
\end{aligned}
$$

Since $A$ is involutive, we have commutation relations coming from the requirement $P_{i j}=P_{j i}$, namely

The trace is given by

$$
\begin{aligned}
C_{i} C_{j}+E_{i} C_{j} C_{2} & =C_{j} C_{i}+E_{j} C_{i} C_{2}, \\
C_{i} E_{j}+E_{i} C_{j} E_{2} & =C_{j} E_{i}+E_{j} C_{i} E_{2}, \\
E_{i} E_{j} & =E_{j} E_{i} .
\end{aligned}
$$

$$
\begin{aligned}
\operatorname{trace}\left(A^{(1)}\right) & =P_{11}+P_{22}+P_{33} \\
& =\sum_{i}\left(\left(C_{i}^{2}+E_{i} C_{i} C_{2}\right) P_{11}+\left(C_{i} E_{i}+E_{i} C_{i} E_{2}\right) P_{12}+E_{i}^{2} P_{22}\right)
\end{aligned}
$$


We must have each coefficient in $\operatorname{tr} A^{(1)}$ identically zero, which gives the following:

$$
\begin{gathered}
C_{1}^{2}+C_{2}^{2}+C_{3}^{2}+E_{2} C_{2}^{2}+E_{3} C_{3} C_{2}=0, \\
C_{2} E_{2}+C_{3} E_{3}+E_{2} C_{2} E_{2}+E_{3} C_{3} E_{2}=0, \\
E_{2}^{2}+E_{3}^{2}=0 .
\end{gathered}
$$

Since we are using Guillemin normal form, we may write our matrices in the following form:

$$
C_{1}=\mathrm{Id}_{s}, \quad C_{2}=\left(\begin{array}{cc}
0 & 0 \\
C_{2}^{\prime} & C_{2}^{\prime \prime}
\end{array}\right), \quad C_{3}=\left(\begin{array}{cc}
C_{3}^{*} & C_{3}^{* *} \\
C_{3}^{\prime} & C_{3}^{\prime \prime}
\end{array}\right),
$$

where $C_{j}^{\prime} \in M_{(s-\kappa) \times \kappa}(\mathbf{R}), C_{j}^{\prime \prime} \in M_{(s-\kappa) \times(s-\kappa)}(\mathbf{R}), C_{3}^{*} \in M_{\kappa \times \kappa}$, and $C_{3}^{* *} \in M_{\kappa \times(s-\kappa)}$.

$$
E_{1}=(0), \quad E_{2}=\left(\begin{array}{cc}
\operatorname{Id}_{\kappa} & 0 \\
0 & 0
\end{array}\right), \quad E_{3}=\left(\begin{array}{cc}
J & 0 \\
0 & 0
\end{array}\right),
$$

where $\operatorname{Id}_{\kappa}, J \in M_{\kappa \times \kappa}(\mathbf{R})$. We also have $J^{2}=-$ Id by (3.6) (or Corollary 2.7). The nontrivial case of (3.2) is $i=3, j=2$ which gives $C_{3} E_{2}+$ $E_{3} C_{2} E_{2}=C_{2} E_{3}+E_{2} C_{3} E_{2}$. Expanding this out, we have

$$
\begin{aligned}
& \left(\begin{array}{ll}
C_{3}^{*} & C_{3}^{* *} \\
C_{3}^{\prime} & C_{3}^{\prime \prime}
\end{array}\right)\left(\begin{array}{ll}
I & 0 \\
0 & 0
\end{array}\right)+\left(\begin{array}{ll}
J & 0 \\
0 & 0
\end{array}\right)\left(\begin{array}{cc}
0 & 0 \\
C_{2}^{\prime} & C_{2}^{\prime \prime}
\end{array}\right)\left(\begin{array}{ll}
I & 0 \\
0 & 0
\end{array}\right) \\
& =\left(\begin{array}{cc}
0 & 0 \\
C_{2}^{\prime} & C_{2}^{\prime \prime}
\end{array}\right)\left(\begin{array}{ll}
J & 0 \\
0 & 0
\end{array}\right)+\left(\begin{array}{ll}
I & 0 \\
0 & 0
\end{array}\right)\left(\begin{array}{cc}
C_{3}^{*} & C_{3}^{* *} \\
C_{3}^{\prime} & C_{3}^{\prime \prime}
\end{array}\right)\left(\begin{array}{ll}
I & 0 \\
0 & 0
\end{array}\right),
\end{aligned}
$$

which gives

$$
C_{3}^{\prime}=C_{2}^{\prime} J
$$

The nontrivial case of (3.1) is $i=3, j=2$ which says

$$
C_{3} C_{2}+E_{3} C_{2}^{2}=C_{2} C_{3}+E_{2} C_{3} C_{2}
$$

which when expanded out gives

$$
\begin{aligned}
& C_{3}^{\prime \prime} C_{2}^{\prime}=C_{2}^{\prime} C_{3}^{*}+C_{2}^{\prime \prime} C_{2}^{\prime} J, \\
& C_{3}^{\prime \prime} C_{2}^{\prime \prime}-C_{2}^{\prime \prime} C_{3}^{\prime \prime}=C_{2}^{\prime} C_{3}^{* *},
\end{aligned}
$$

where we have used (3.8) to eliminate $C_{3}^{\prime}$. Similarly, expanding (3.4) yields

$$
\begin{gathered}
I+C_{3}^{* 2}+C_{3}^{* *} C_{2}^{\prime} J+J C_{3}^{* *} C_{2}^{\prime}=0 \\
C_{3}^{*} C_{3}^{* *}+C_{3}^{* *} C_{3}^{\prime \prime}+J C_{3}^{* *} C_{2}^{\prime \prime}=0 \\
I+C_{2}^{\prime \prime 2}+C_{2}^{\prime} J C_{3}^{* *}+C_{2}^{\prime \prime 2}=0
\end{gathered}
$$


while (3.5) implies

$$
C_{3}^{*} J+J C_{3}^{*}=0
$$

and we have already adjusted $E_{3}$ such that (3.6) holds.

In summary we look for matrices $C_{2}^{\prime}, C_{2}^{\prime \prime}, C_{3}^{*}, C_{3}^{* *}, C_{3}^{\prime \prime}$ and a complex structure $J$ satisfying (3.10)-(3.15). We would like to use the $\mathrm{Gl}(s, \mathbf{R}) \times$ $\mathrm{SO}(3)$ equivalence to make these equations simpler but we do not want to break Guillemin normal form.

On the other hand, if we normalize by

$$
g=\left(\begin{array}{cc}
I_{\kappa} & X \\
0 & I_{s-\kappa}
\end{array}\right) \in \mathrm{Gl}(s, \mathbf{R}),
$$

Guillemin normal form is preserved, and

$$
\begin{aligned}
& J \rightarrow J, \\
& C_{3}^{*} \rightarrow C_{3}^{*}+X C_{2}^{\prime} J-J X C_{2}^{\prime}, \\
& C_{3}^{* *} \rightarrow C_{3}^{* *}-C_{3}^{*} X-X C_{2}^{\prime} J X-J X C_{2}^{\prime} X+J C_{2}^{\prime \prime}+X C_{3}^{\prime \prime}, \\
& C_{2}^{\prime} \rightarrow C_{2}^{\prime}, \\
& C_{2}^{\prime \prime} \rightarrow C_{2}^{\prime \prime}-C_{2}^{\prime} X, \\
& C_{3}^{\prime \prime} \rightarrow C_{3}^{\prime \prime}-C_{2}^{\prime} J X .
\end{aligned}
$$

If we normalize by

$$
g=\left(\begin{array}{cc}
A & 0 \\
0 & B
\end{array}\right) \in \mathrm{Gl}(s, \mathbf{R}), \quad A \in \mathrm{Gl}(\kappa, \mathbf{R}), \quad B \in \mathrm{Gl}(s-\kappa, \mathbf{R}),
$$

Guillemin normal form is also preserved and

$$
\begin{aligned}
& J \rightarrow A J A^{-1}, \\
& C_{3}^{*} \rightarrow A C_{3}^{*} A^{-1}, \\
& C_{3}^{* *} \rightarrow A C_{3}^{* *} B^{-1}, \\
& C_{2}^{\prime} \rightarrow B C_{2}^{\prime} A^{-1}, \\
& C_{2}^{\prime \prime} \rightarrow B C_{2}^{\prime \prime} B^{-1}, \\
& C_{3}^{\prime \prime} \rightarrow B C_{3}^{\prime \prime} B^{-1} .
\end{aligned}
$$

If we tried to normalize by $g=\left(\begin{array}{cc}I_{\kappa} & 0 \\ Y & I_{s-\kappa}\end{array}\right) \in \mathrm{Gl}(s, \mathbf{R})$, Guillemin normal form would be broken. Similarly we may only normalize by an $\mathrm{SO}(2) \subset$ $\mathrm{SO}(3)$ action (the $\mathrm{SO}(2)$ that fixes the first column) without breaking Guillemin normal form and this action changes things rather drastically, making it difficult to use except in special cases. 
We now prove Theorem 3.1, i.e., we classify the case $\kappa=2$.

In this case we use $A$ in (3.18) to normalize $J$ to be the standard complex structure $\left(\begin{array}{cc}0 & -1 \\ 1 & 0\end{array}\right)$. Note that (3.15) now implies that $C_{3}^{*}$ is traceless symmetric.

Case 1. $s=s_{1}$ is odd.

Case 1.0. $C_{2}^{\prime}=0$. Here (3.12) implies that $C_{3}^{* 2}=-I_{2}$, which is a contradiction because $C_{3}^{* 2}$ must have positive eigenvalues by (3.15).

Case 1.1. rank $C_{2}^{\prime}=1$. Use $A$ in (3.18) to get

$$
C_{3}^{*}=\left(\begin{array}{cc}
x & 0 \\
0 & -x
\end{array}\right) \text {, }
$$

which can be done by an element of $\mathrm{Gl}(1, \mathbf{C}) \subset \mathrm{Gl}(2, \mathbf{R})$ (and thus leaving $J$ unchanged) again because of (3.15).

Use $B$ in (3.18) to get

$$
C_{2}^{\prime}=\left(\begin{array}{cc}
1 & u \\
0 & 0 \\
\vdots & \vdots \\
0 & 0
\end{array}\right)
$$

where $u$ is unknown.

Use $X$ in (3.16) to get

$$
C_{2}^{\prime \prime}=\left(\begin{array}{cc}
0 & 0 \\
f_{2} & F_{2}
\end{array}\right), \quad C_{3}^{\prime \prime}=\left(\begin{array}{cc}
0 & 0 \\
f_{3} & F_{3}
\end{array}\right),
$$

where $f_{j} \in M_{(s-3) \times 1}$ and $F_{j} \in M_{(s-3) \times(s-3)}$. This can be done because the top rows of $C_{2}^{\prime} X$ and $C_{2}^{\prime} J X$ are linearly independent (regardless of what $u$ is).

Now (3.10) becomes

$$
\begin{aligned}
\left(\begin{array}{cc}
0 & 0 \\
f_{3} & F_{3}
\end{array}\right)\left(\begin{array}{ll}
1 & u \\
0 & 0
\end{array}\right)= & \left(\begin{array}{ll}
1 & u \\
0 & 0
\end{array}\right)\left(\begin{array}{cc}
x & 0 \\
0 & -x
\end{array}\right) \\
& +\left(\begin{array}{cc}
0 & 0 \\
f_{2} & F_{2}
\end{array}\right)\left(\begin{array}{cc}
1 & u \\
0 & 0
\end{array}\right)\left(\begin{array}{cc}
0 & -1 \\
1 & 0
\end{array}\right),
\end{aligned}
$$

which implies $x=0$ and $f_{2}=f_{3}=0$. Now that we know $C_{3}^{*}=0$ we are free again to use $A$ to make $u=0$. Write

Then (3.11) gives

$$
C_{3}^{* *}=\left(\begin{array}{ccc}
r_{1}^{1} & \cdots & r_{s-2}^{1} \\
r_{1}^{2} & \cdots & r_{s-2}^{2}
\end{array}\right)
$$

$$
\left(\begin{array}{cc}
0 & 0 \\
0 & F_{3}
\end{array}\right)\left(\begin{array}{cc}
0 & 0 \\
0 & F_{2}
\end{array}\right)-\left(\begin{array}{cc}
0 & 0 \\
0 & F_{2}
\end{array}\right)\left(\begin{array}{cc}
0 & 0 \\
0 & F_{3}
\end{array}\right)=\left(\begin{array}{ll}
1 & 0 \\
0 & 0
\end{array}\right)\left(\begin{array}{ccc}
r_{1}^{1} & \cdots & r_{s-2}^{1} \\
r_{1}^{2} & \cdots & r_{s-2}^{2},
\end{array}\right),
$$


which implies

$$
r_{1}^{1}=\cdots=r_{s-2}^{1}=0, \quad\left[F_{3}, F_{2}\right]=0 .
$$

Finally (3.14) gives

$$
\left(\begin{array}{ll}
1 & 0 \\
0 & I
\end{array}\right)+\left(\begin{array}{cc}
0 & 0 \\
0 & F_{2}^{2}
\end{array}\right)+\left(\begin{array}{cc}
0 & 0 \\
0 & F_{3}^{2}
\end{array}\right)=\left(\begin{array}{ll}
1 & 0 \\
0 & 0
\end{array}\right)\left(\begin{array}{ccc}
-r_{1}^{2} & \cdots & -r_{s-2}^{2} \\
0 & \cdots & 0
\end{array}\right),
$$

which implies $r_{2}^{2}=\cdots=r_{s-2}^{2}=0, r_{1}^{2}=-1$, and

$$
I+F_{2}^{2}+F_{3}^{2}=0 .
$$

This shows the $\mathrm{m}$-tableau is reducible in the sense that the top three rows do not interact with the bottom $s-3$ rows (thinking in terms of equations, the first three equations form an independent system from the others). In fact we may recognize the top m-tableau to be the special Lagrangian $m$ tableau while the bottom is a Cauchy-Riemann m-tableau by Lemma 2.4. We see any involutive m-tableau in this case is $\mathrm{Gl}(s, \mathbf{R}) \times \mathrm{SO}(3)$ equivalent to

$$
\left(\begin{array}{c}
\text { SLag } \\
\text { CR }
\end{array}\right)=\left(\begin{array}{ccc}
\pi_{1}^{1} & \pi_{2}^{1} & -\left(\pi_{1}^{3}+\pi_{2}^{2}\right) \\
\pi_{1}^{2} & \pi_{2}^{2} & \pi_{2}^{1} \\
\pi_{1}^{3} & \pi_{1}^{2} & \pi_{1}^{1} \\
\Pi & J_{s-3} \Pi & 0
\end{array}\right),
$$

where $J_{s-3}$ is any complex structure on $\mathbf{R}^{s-3}$ and

$$
\Pi=\left(\begin{array}{c}
\pi_{1}^{4} \\
\vdots \\
\pi_{1}^{s}
\end{array}\right) \text {. }
$$

Case 1.2. rank $C_{2}^{\prime}=2$. Use $A$ and $B$ in (3.18) to get

$$
C_{3}^{*}=\left(\begin{array}{cc}
x & 0 \\
0 & -x
\end{array}\right), \quad C_{2}^{\prime}=\left(\begin{array}{c}
I_{2} \\
0
\end{array}\right) \text {. }
$$

Write

$$
X=\left(\begin{array}{lll}
x_{1} & \cdots & x_{s-2} \\
y_{1} & \cdots & y_{s-2}
\end{array}\right)
$$

Use $X$ of (3.16) to get $C_{3}^{*}=0$.

Since

$$
X C_{2}^{\prime} J-J X C_{2}^{\prime}=\left(\begin{array}{cc}
x_{2}+y_{1} & y_{2}-x_{1} \\
y_{2}-x_{1} & -\left(x_{2}+y_{1}\right),
\end{array}\right),
$$

the remaining freedom in $X$ after normalizing $C_{3}^{*}$ to be zero is

$$
X=\left(\begin{array}{ccccc}
\lambda & -\mu & x_{3} & \cdots & x_{s-2} \\
\mu & \lambda & y_{3} & \cdots & y_{s-2}
\end{array}\right) \text {. }
$$


We use this to get

$$
C_{2}^{\prime}=\left(\begin{array}{cc}
a_{2} & 0 \\
f_{2} & F_{2}
\end{array}\right)
$$

where $a_{2}$ is $2 \times 2, F_{2}$ is $(s-4) \times(s-4)$, and $a_{2} J+J a_{2}=0$.

Now (3.10) implies $a_{3}=a_{2} J, f_{3}=f_{2} J$. Writing $C_{3}^{* *}=\left(\begin{array}{ll}R_{1} & R_{2}\end{array}\right)$, where $R_{1}$ is $2 \times 2,(3.11)$ gives

$$
-2 a_{2}^{2} J+g_{3} f_{2}=R_{1} \text {. }
$$

Substituting for $g_{3} f_{2}$ in (3.14) gives $J R_{1}=0$. This implies $R_{1}=0$, but (3.12) says $R_{1} J+J R_{1}=-\mathrm{Id}_{2}$, giving a contradiction.

Case 2. $s$ is even (Note that $s=2$ cannot occur because $\mathbf{R}^{2}$ is not a $\mathrm{Cl}_{2}$ module, so assume $s \geq 4$.)

Case 2.0. $C_{2}^{\prime}=(0)$. Again (3.12) implies $C_{3}^{* 2}=-I_{2}$ giving a contradiction.

Case 2.1. rank $C_{2}^{\prime}=1$. Nothing about $s_{1}$ being odd was used to derive the splitting of the m-tableau in Case 1.1, but now $s-3$ is odd and Lemma 2.9 gives a contradiction.

Case 2.2. rank $C_{2}^{\prime}=2$. Use $B$ in (3.18) to get

$$
C_{2}^{\prime}=\left(\begin{array}{c}
I_{2} \\
0
\end{array}\right)
$$

and $X$ in (3.16) to get

$$
\begin{gathered}
C_{2}^{\prime \prime}=\left(\begin{array}{cc}
0 & 0 \\
f_{2} & F_{2}
\end{array}\right), \quad C_{3}^{\prime \prime}=\left(\begin{array}{cc}
0 & 0 \\
f_{3} & F_{3}
\end{array}\right), \\
f_{j} \in M_{(s-3) \times 1}, \quad F_{j} \in M_{(s-3) \times(s-3)} .
\end{gathered}
$$

This can be done because the top rows of $C_{2}^{\prime} X$ and $C_{2}^{\prime} J X$ are respectively the first row and minus the second row of $X$.

(3.11) gives

$$
\begin{gathered}
\left(\begin{array}{cc}
0 & 0 \\
f_{3} & F_{3}
\end{array}\right)\left(\begin{array}{cc}
0 & 0 \\
f_{2} & F_{2}
\end{array}\right)-\left(\begin{array}{cc}
0 & 0 \\
f_{2} & F_{2}
\end{array}\right)\left(\begin{array}{cc}
0 & 0 \\
f_{3} & F_{3}
\end{array}\right) \\
=\left(\begin{array}{c}
I_{2} \\
0
\end{array}\right)\left(\begin{array}{lll}
r_{1}^{1} & \cdots & r_{s-2}^{1} \\
r_{1}^{2} & \cdots & r_{s-2}^{2}
\end{array}\right),
\end{gathered}
$$

which implies

$$
r_{1}^{1}=\cdots=r_{s-2}^{1}=0 \text {. }
$$

Finally (3.14) gives, using (3.25),

$$
\left(\begin{array}{ll}
1 & 0 \\
0 & I
\end{array}\right)+\left(\begin{array}{cc}
0 & 0 \\
0 & F_{2}^{2}
\end{array}\right)+\left(\begin{array}{cc}
0 & 0 \\
0 & F_{3}^{2}
\end{array}\right)=\left(\begin{array}{ccc}
-r_{1}^{2} & \cdots & -r_{s-2}^{2} \\
0 & \cdots & 0
\end{array}\right),
$$


which implies

$$
I+F_{2}^{2}+F_{3}^{2}=0
$$

and $r_{2}^{2}=\cdots=r_{s-2}^{2}=0$. Substituting the zero $r$ values into (3.24) we see

$$
\left[F_{3}, F_{2}\right]=0 \text {. }
$$

Now (3.28) says that $F_{2}$ and $F_{3}$ share common eigenspaces, in particular, since they are odd dimensional they share a common real eigenspace, thus their squares have a common eigenspace with nonnegative eigenvalue, which gives a contradiction by (3.27). This concludes the proof of Theorem 3.1. q.e.d.

We now prove Theorem 3.2. If $C_{2}^{\prime}=0$ then (3.14) gives a contradiction, so assume $C_{2}^{\prime} \neq 0$. Use $X$ of (3.16) to get $C_{2}^{\prime \prime}=C_{3}^{\prime \prime}=0$, and fix $J=\left(\begin{array}{cc}0 & - \text { Id } \\ \text { Id } & 0\end{array}\right)$. Use $A$ of (3.18) to get

$$
C_{3}^{*}=\left(\begin{array}{cc}
m & 0 \\
0 & -m
\end{array}\right) \text {, }
$$

where $m$ is $\frac{\kappa}{2} \times \frac{\kappa}{2}$. We may use the remaining freedom in $A$ to get

$$
C_{2}^{\prime}=\left(\begin{array}{lllll}
r & 0 & \cdots & 0 & \delta
\end{array}\right)=\left(\begin{array}{ll}
\gamma & \delta
\end{array}\right),
$$

where $\gamma, \delta$ are rows of length $\frac{\kappa}{2}$.

Write $C_{3}^{* *}=\left(\begin{array}{c}\alpha \\ \beta\end{array}\right)$. In this notation the equations are

$$
\begin{gathered}
\gamma m=-\delta m=0, \\
\gamma \alpha+\delta \beta=0, \\
\mathrm{Id}+m^{2}+\alpha \delta-\beta \gamma=0, \\
\alpha \gamma+\beta \delta=0, \\
m \alpha=m \beta=0, \\
1-\gamma \beta+\delta \alpha=0 .
\end{gathered}
$$

Now (3.12.2') implies $\beta=\lambda \alpha$ and $\delta=-\frac{1}{\lambda} \gamma$ for some $\lambda \in \mathbf{R}$. This is because in indices it says $\alpha^{j} \gamma_{i}+\beta^{j} \delta_{i}=0 \forall i, j$. Fixing $i=1$ we get $\beta=\lambda \alpha$ and since both $\alpha, \delta$ cannot both be zero we fix some $j$ to get $\delta=-\frac{1}{\lambda} \gamma$.

$\left(3.10^{\prime}\right)$ implies

$$
m=\left(\begin{array}{cc}
0 & 0 \\
\hat{m} & \tilde{m}
\end{array}\right)
$$

where $\hat{m}$ is a column and $\tilde{m}$ is $\frac{\kappa}{2} \times \frac{\kappa}{2} \cdot\left(3.12 .1^{\prime}\right)$ now says $\tilde{m}^{2}=-$ Id so, in particular, it has no kernel. 
$\left(3.13^{\prime}\right)$ says

$$
\tilde{m}\left(\begin{array}{c}
\alpha^{2} \\
\vdots \\
\alpha^{\frac{\kappa}{2}}
\end{array}\right)=0
$$

so $\left(3.12 .1^{\prime}\right)$ implies

$$
\alpha=\left(\begin{array}{c}
\alpha^{1} \\
0 \\
\vdots \\
0
\end{array}\right)
$$

Writing this all out we have

$$
\begin{aligned}
& C_{2}=\left(\begin{array}{ccccc}
0 & 0 & 0 & 0 & 0 \\
0 & 0 & 0 & 0 & 0 \\
0 & 0 & 0 & 0 & 0 \\
0 & 0 & 0 & 0 & 0 \\
r & 0 & -\frac{r}{\lambda} & 0 & 0
\end{array}\right), \quad C_{3}=\left(\begin{array}{ccccc}
0 & 0 & 0 & 0 & \alpha \\
\hat{m} & \tilde{m} & 0 & 0 & 0 \\
0 & 0 & 0 & 0 & \lambda \alpha^{1} \\
0 & 0 & \hat{m} & \tilde{m} & 0 \\
\frac{r}{\lambda} & 0 & r & 0 & 0
\end{array}\right), \\
& E_{3}=\left(\begin{array}{ccccc}
0 & 0 & -1 & 0 & 0 \\
0 & 0 & 0 & - \text { Id } & 0 \\
1 & 0 & 0 & 0 & 0 \\
0 & \text { Id } & 0 & 0 & 0 \\
0 & 0 & 0 & 0 & 0
\end{array}\right),
\end{aligned}
$$

where the blocking is of the form $\left(1, \frac{\kappa}{2}, 1, \frac{\kappa}{2}, 1\right) \times\left(1, \frac{\kappa}{2}, 1, \frac{\kappa}{2}, 1\right)$. We see that the first, $\frac{\kappa}{2}$ th, and last rows do not interact with the others and we know from the previous classification that the pieces must be special Lagrangian and H-Dirac type m-tableaux, concluding the proof of Theorem 3.2. q.e.d.

The preceding discussion covers all of the cases of Theorem 3.3 except for the proof of the $4 \times 5,5 \times 5$, and $5 \times 4$ cases, which are similar to the above and left to the reader.

\section{PART II: EXAMPLES AND RIGIDITY OF M-SUBSETS}

We now address the question of finding first-order systems implying the minimal submanifold system. First we rephrase the question geometrically; identifying m-tableaux with candidates for tangent spaces to m-subsets. By the results of Part I, this immediately gives new restrictions on the dimensions of $m$-subsets. Next, for various $m$-tableaux, we analyze the corresponding differential systems for m-subsets, i.e., we see 
what linear first-order systems from Part I are actually the linearization of a first-order system implying the minimal submanifold system. The results are rather striking. In cases of dimension or codimension two, and certain other Cauchy-Riemann m-tableaux, there are abundant examples of m-subsets with a given type of m-tableau. For other m-tableaux, including all the other standard examples of m-tableaux, we show rigidity in the sense that any m-subset having tangent spaces isomorphic to the given m-tableau must be the standard face corresponding to that m-tableau.

\section{1. $\mathbf{m}$-tableaux as candidate tangent spaces to $\mathbf{m}$-subsets}

Given $f: M^{n} \rightarrow \mathbf{R}^{n+s}$ any (oriented) submanifold, we have the Gauss map $\gamma_{f}: M \rightarrow G_{n, n+s}$ defined by $\gamma_{f}(x)=T_{f(x)} f(M)$, where $G_{n, n+s}$ denotes the (oriented) Grassmannian of $n$-planes in $\mathbf{R}^{n+s}$.

Let $E \in G_{n, n+s}$. We have the identification $T_{E} G_{n, n+s} \simeq E^{\perp} \otimes E^{*}$, where $E^{\perp}$ denotes the orthogonal complement of $E$ in $\mathbf{R}^{n+s}$. If $g$ : $M \rightarrow G_{n, n+s}$ is any map, then for all $x \in M, g_{* x}$ may be considered as an element of $E^{\perp} \otimes E^{*} \otimes T_{x}^{*}$. If $g=\gamma_{f}$, then $T_{x}=E$ and in fact $\gamma_{f *} \in E^{\perp} \otimes \operatorname{Sym}^{2} E^{*}$, essentially because mixed partials commute.

The derivative of the Gauss map $\gamma_{f_{*}}$ is called the second fundamental form. The minimal submanifold condition is that $\gamma_{f *}$ be traceless, i.e., $\gamma_{f *} \in E^{\perp} \otimes \operatorname{Sym}_{0}^{2} E^{*}$ at each point of $M$.

Let $\Sigma \subset G_{n, n+s}$ and denote $T_{E} \Sigma$ by $A . A$ is a linear subspace of $E^{\perp} \otimes$ $E^{*}$. What are the possible second fundamental forms of submanifolds $f(M)$ such that $\gamma_{f}(M) \subset \Sigma$ ? The derivative at $g(x)=E \in \Sigma$ of any map $g: M \rightarrow \Sigma$ is a linear map $g_{* x}: T_{x} M \rightarrow A$ and we think of $g_{* x}$ as an element of the linear space $A \otimes T_{x}^{*}$. If the map is a second fundamental form, so $E=T_{x} M$, then $g_{* x}$ must also lie in $E^{\perp} \otimes \operatorname{Sym}^{2} E^{*}$. So let

$$
A^{(1)}:=\left(A \otimes E^{*}\right) \cap\left(E^{\perp} \otimes \operatorname{Sym}^{2} E^{*}\right) \text {. }
$$

This is the space of all candidate second fundamental forms at $E$ for submanifolds $f(M)$ having Gauss map locally contained in $\Sigma$.

Fundamental observation. If $\Sigma \subset G_{n, n+s}$ is such that for all $E \in \Sigma$, $\left(T_{E} \Sigma\right)^{(1)}$ is traceless, then $\Sigma$ is an m-subset.

Remark. When we consider $A$ as a tableau, $A^{(1)}$ is just the prolongation of $A$ as defined in Part I, $\S 1$.

So we may rephrase the observation by saying that $\Sigma$ is an m-subset if all its tangent planes are m-tableaux. It is in this sense that linear 
first-order systems implying the Laplace system, considered as m-tableaux, are candidate tangent spaces to m-subsets.

Examples. In [8], Harvey and Lawson study the following m-subsets (faces) arising from calibrations:

- The $\mathrm{SU}(n)$ orbit of a real $n$-plane in $\mathbf{R}^{2 n}$, called the special Lagrangian face, $\operatorname{SLag}_{n, 2 n}$. It has m-tableau as in Example $2^{\prime}$ of Part I.

- The $G_{2}$ orbit of an associative 3-plane in $\mathbf{R}^{7}$, called the associative face. It has m-tableau corresponding to the Dirac operator in Example $3^{\prime}$ of Part I, given in coordinates by I.2.1 with $n=3, s=4$.

- The $G_{2}$ orbit of a coassociative 4-plane in $\mathbf{R}^{7}$, called the coassociative face. Its $\mathrm{m}$-tableau is the transpose of the associative $\mathrm{m}$-tableau and as an operator it is the formal adjoint of the operator corresponding to the associative m-tableau.

- The $\operatorname{Spin}(7)$ orbit of a quaternionic 4-plane in $\mathbf{R}^{8}$, called the Cayley face. It has $m$-tableau corresponding to the Dirac operator in Example $3^{\prime}$ of Part I, given in coordinates by I.2.1 with $n=4, s=4$.

- Of course the face of a normalized power of the Kähler form is just the complex Grassmannian $G\left(\mathbf{C}^{n}, \mathbf{C}^{n+s}\right) \subset G_{2 n, 2 n+2 s}$ which is the $\mathrm{SU}(n+s)$ orbit of a complex $n$-plane.

Remark. It is clear for global reasons that these faces of calibrations must be m-subsets, but the reader may find it interesting to try to show this directly.

Remark. These faces all have involutive m-tableaux. (When necessary, we will call $\mathrm{m}$-subsets whose $\mathrm{m}$-tableaux are involutive, involutive $m$-subsets.) Faces of calibrations with noninvolutive m-tableaux are also studied. They have a small class of calibrated manifolds as we would expect by the remark on noninvolutive tableaux in Part I. Their utility, first noticed by F. Morgan, is that they often calibrate manifolds with singularities which were previously only suspected to be area minimizing. For example, D. Nance, in [13], uses a noninvolutive face to prove the sufficiency of Morgan's Angle Criterion for a pair of planes to be area minimizing.

At first glance m-subsets are not of much use in studying area minimizing submanifolds with singularities. However singular manifolds often appear as the simplest $\Sigma$-manifolds (in the sense that they correspond to the simplest solutions to the differential system induced by an m-subset $\Sigma)$. These singular $\Sigma$-manifolds are good candidates for area minimizing submanifolds. For example, the $\Sigma$-manifolds produced by Theorem 3.9 are examples of such candidates, one class of which is proved to be locally minimizing in the appendix. 
It was known (e.g., [5]) that $\mathrm{SLag}_{3,6}$, the associative, coassociative, and Cayley faces, $G\left(\mathbf{C}, \mathbf{C}^{m+1}\right)$, and $G\left(\mathbf{C}^{m}, \mathbf{C}^{m+1}\right)$ are maximal as faces, i.e., that they are not contained in any larger faces in their respective Grassmannian. From the results of Part I we see that they are also m-subsets of maximal dimension in their respective Grassmannians, i.e., there is no larger dimensional $\mathrm{m}$-subset in their respective Grassmannians. Using Theorems I.3.1, I.3.2, and I.3.3 it is possible to add to this list.

Theorem 1.1. The following involutive $m$-subsets are of maximal dimension:

1. $\mathrm{SLag}_{5,10} \subset G_{5,10}$.

2. The product $G\left(\mathbf{C}, \mathbf{C}^{\left(\frac{s+2)}{2}\right)} \times \mathrm{SLag}_{3,6} \subset G_{5, s+5}\right.$ of a complex Grassmannian $G\left(\mathbf{C}, \mathbf{C}^{\frac{(s+2)}{2}}\right)$ in $G_{2, s+2}$ with a special Lagrangian face $\mathrm{SLag}_{3,6}$ in $G_{3,6}$.

3. $A$ Cayley face in $G_{4,9}$.

4. A Cayley face in $G_{5,9}$.

5. $G\left(\mathbf{C}^{m}, \mathbf{C}^{m+1}\right) \subset G_{2 m+1,2 m+3}$.

In the remainder of this part we study what possible m-subsets can occur. We determine how many, if any, first-order systems implying the minimal submanifold system correspond to various m-tableaux.

\section{Setting up the problem}

We now set up the differential systems for $\mathrm{m}$-subsets having a given $\mathrm{m}$ tableau $A$. By this we mean that we look for $\Sigma$ having $T_{E} \Sigma \sim A \forall E \in \Sigma$, where ' $\sim$ ' means

$$
T_{[g]} \Sigma \in L_{g *}(\mathrm{Gl}(s, \mathbf{R}) \times \mathrm{SO}(n) \text { orbit of } A)
$$

(i.e., if $A$ has dimension $a$, we want $T_{\text {[Id] }} \Sigma$ to be an element of the $\mathrm{Gl}(s, \mathbf{R}) \times \mathrm{SO}(n)$ orbit of $A$ in the Grassmanian $G\left(a, T_{[\mathrm{Id}]} G_{n, n+s}\right)$, and $T_{[g]} \Sigma$ to be an element of the translate of this orbit to an orbit in $G\left(a, T_{[g]} G_{n, n+s}\right)$. Here $G_{n, n+s}$ is being considered as a homogeneous space of $\mathrm{SO}(n+s), g \in \mathrm{SO}(n+s), L_{g *}$ denotes the derivative of left action by $g$ and $[g]=E$. Since smooth m-subsets have the same type of $m$-tableaux in small neighborhoods, this is no restriction for the local problem of finding smooth $\mathrm{m}$-subsets and smooth minimal submanifolds.

To set up the appropriate differential systems, we want to allow $T_{E} \Sigma$ to be equivalent to anything in the $\mathrm{Gl}\left(E^{\perp}\right) \times \mathrm{SO}(E)$ orbit of $L_{g *} A$. To 
make this precise we will set up a more general situation.

Let $G / H$ be a homogeneous space. Write $\mathfrak{m}=\mathfrak{g} / \mathfrak{h}$ and let $K$ be a group that acts linearly on $\mathfrak{m}$. Let $A$ be a given $p$-dimensional subspace of $\mathfrak{m}$. We will define a differential system for $p$-dimensional submanifolds of $G / H$ whose tangent spaces are $K$-equivalent to $A$, i.e., we will set up a differential system whose solutions are $\Sigma^{p} \subset G / H$ such that

for some $k \in K$.

$$
T_{[g]} \Sigma=L_{g *}\left(k^{-1} A\right)
$$

(The case that will be studied in this part is $G / H=G_{n, n+s}, K=$ $\mathrm{GL}(s, \mathbf{R}) \times \mathrm{SO}(n), A$ : an m-tableau. $)$ We will set up the system on $G \times K$ as there is a convenient coframing there by Maurer-Cartan forms. Solutions of the system on $G \times K$ will be lifts of solutions of the differential system on $G / H$ for submanifolds having tangent spaces $K$-equivalent to A.

Consider the map

$$
\begin{aligned}
& G \times K \stackrel{f_{A}}{\longrightarrow} G_{n}(T(G / H)), \\
& (g, k) \mapsto\left([g], L_{g *}\left(k^{-1} A\right)\right),
\end{aligned}
$$

where $G_{n}(T(G / H))$ denotes the Grassmann bundle of all $n$-planes in all tangent spaces of $G / H$. The system we want is just the pullback via $f_{A}$ of the canonical system on $G_{n}(T(G / H))$. At $\left(x, E_{x}^{n}\right) \in G_{p}(T(G / H))$, the canonical system is

$$
\mathscr{I}=\pi^{*}\left(E^{\perp}\right), \quad \mathscr{J}=\pi^{*}\left(T_{x}^{*}(G / H)\right),
$$

where $\pi$ is the projection $G_{n}(T(G / H)) \rightarrow G / H$, and $E^{\perp}$ denotes the forms in $T_{x}^{*}(G / H)$ annihilating $E$. (See [4, Chapter IV] for more details on the canonical system on the Grassmann bundle of a manifold.)

Assume we have a metric on $\mathfrak{m}$ giving rise to a left invariant metric on $G / H$. (If $G$ is compact as in our case, there is a natural metric.) Let $\left\{v^{i *}\right\}$ be an orthonormal basis of $\mathrm{m}^{*}$.

Let $\gamma_{i}$ be the unique left invariant one-form on $G / H$ which is equal to $v^{i *}$ at $[e]$. (Thinking of $\mathfrak{g}=\mathfrak{h} \oplus \mathfrak{m}$, we may consider $\gamma$ as an $\mathfrak{m}$ valued one-form.)

Say $A$ is given by equations $b_{i}^{r} v^{i}=0,1 \leq r \leq \operatorname{codim}(A)$. This means $A^{\perp} \subset \mathrm{m}^{*}$ has basis $\left\{b_{i}^{r} v^{i *}\right\}$. Then in coordinates, $\left.f_{A}^{*}(\mathscr{I})\right|_{(g, k)}$ has generators $k^{-1} L_{g *} b_{i}^{r} v^{i *}=k^{-1}\left(b_{i}^{r} \gamma_{i}\right)$.

Following this general procedure in our case of the system for m-subsets with a given m-tableau, one would naively set up a system on $\mathrm{Gl}(s) \times$ $\mathrm{SO}(n+s) \times \mathrm{SO}(n)$, but here the $\mathrm{SO}(n)$ factor is unnecessary because it 
already is incorporated in $\mathrm{SO}(n+s)$. In fact most of the vector fields on $\mathrm{GL}(s)$ will be Cauchy characteristics, so we work on $\mathrm{Gl}(s) \times \mathrm{SO}(n+s)$ and expect at least $\left(\begin{array}{l}s \\ 2\end{array}\right)+1$ Cauchy characteristics related to the $\mathrm{GL}(s)$ action.

For $g \in \mathrm{SO}(n+s)$ write

$$
g^{-1} d g=\left(\begin{array}{cc}
\tau & -{ }^{t} \phi \\
\phi & \tilde{\delta}
\end{array}\right)
$$

For $B \in \mathrm{GL}(s)$, let

$$
\beta=B^{-1} d B, \quad \psi=B^{-1} \phi, \quad \delta=B^{-1} \tilde{\delta} B, \quad \kappa=\beta+\delta .
$$

Notice that $\kappa \in \mathfrak{g l}(s)$ and $\tau \in \mathfrak{s o}(n) . \phi$ is semibasic to the projection $\mathrm{SO}(n+s) \rightarrow G_{n, n+s}$ and it already has an $\mathrm{SO}(s) \times \mathrm{SO}(n)$ action built into it, but we use $\psi$, which is left invariant under all of $K .\left(\psi=k^{-1} \gamma\right.$ in the notation of the general setting.)

We have the following structure equations:

$$
\begin{aligned}
d \psi= & -\left(B^{-1} d B B^{-1}\right) \wedge \phi+B^{-1}(-\phi \wedge \tau-\tilde{\delta} \wedge \phi) \\
= & -\kappa \wedge \psi-\psi \wedge \tau, \\
d \tau= & -\tau \wedge \tau+{ }^{t} \phi \wedge \phi=-\tau \wedge \tau+{ }^{t} \psi^{t} B B \wedge \psi, \\
d \kappa= & -\beta \wedge \beta-\left(-B^{-1} d B B^{-1}\right) \wedge \tilde{\delta} B+B^{-1}\left(\phi \wedge{ }^{t} \phi-\tilde{\delta} \wedge \tilde{\delta}\right) B \\
& +B^{-1} \tilde{\delta} \wedge d B \\
= & -\kappa \wedge \kappa+\psi \wedge^{t} \psi^{t} B B .
\end{aligned}
$$

The independence condition for the differential system is given by

$$
\Omega=\psi_{1}^{1} \wedge \cdots \wedge \psi_{1}^{s_{1}} \wedge \psi_{2}^{1} \wedge \cdots \wedge \psi_{2}^{s_{2}} \wedge \cdots \wedge \psi_{p}^{1} \wedge \cdots \wedge \psi_{p}^{s_{p}},
$$

where we assume $A$ is in Cartan normal form, i.e., that we have chosen bases such that there are no relations involving only $A_{1}^{1}, \cdots, A_{1}^{s_{1}}, A_{2}^{1}, \cdots$, $A_{2}^{s_{2}}, \cdots, A_{p}^{1}, \cdots, A_{p}^{s_{p}}$, where $s_{p}$ is the character of the system. Let the relations on $A$ be $b_{a}^{r i} A_{i}^{a}=0,1 \leq r \leq n s-\left(s_{1}+\cdots+s_{p}\right)$. Let $\theta^{r}=b_{a}^{r i} \psi_{i}^{a}$. Then the differential ideal $\mathscr{I}$ of forms which vanish on solutions is generated by $\left\{\theta^{r}\right\}$. The m-tableaux we will deal with will often have symmetries and we will adapt our notation to utilize them.

\section{Clifford m-subsets and their duals}

This section classifies a large class of $\mathrm{m}$-subsets including the $\mathrm{m}$-subsets corresponding to linear systems involving the fewest possible equations, the Dirac m-tableaux (see equation (I.2.1)). 
Example. $m$-subsets having the Cauchy-Riemann m-tableau in $G_{2, s+2}$. We have

$$
A=(\pi, J \pi), \quad \text { where } \pi=\left(\begin{array}{c}
\pi^{1} \\
\vdots \\
\pi^{s}
\end{array}\right), J^{2}=- \text { Id. }
$$

Take $\mathscr{I}=\left\{\theta:=\psi_{2}-J \psi_{1}\right\}$ with independence condition $\Omega=\psi_{1}^{1} \wedge$ $\cdots \wedge \psi_{1}^{s}$ where

$$
\psi_{j}=\left(\begin{array}{c}
\psi_{j}^{1} \\
\vdots \\
\psi_{j}^{s}
\end{array}\right) \quad \text { and } \quad \theta=\left(\begin{array}{c}
\theta^{1} \\
\vdots \\
\theta^{s}
\end{array}\right)
$$

Taking derivatives, we have

$$
\begin{aligned}
d \theta= & -\kappa \wedge \psi_{2}-\psi \wedge \tau_{2}+J\left(\kappa \psi_{1}+\psi \wedge \tau_{1}\right) \\
\equiv & -\kappa \wedge J \psi_{1}-\left(\psi_{1}, J \psi_{1}\right) \wedge \tau_{2}+J \kappa \wedge \psi_{1} \\
& +\left(J \psi_{1},-\psi_{1}\right) \wedge \tau_{1} \bmod \mathscr{I} \\
\equiv & {[J, \kappa] \wedge \psi_{1}+\left(\tau_{2}^{1}+\tau_{1}^{2}\right) \wedge \psi_{1} \bmod \mathscr{I} } \\
\equiv & {[J, \kappa] \wedge \psi_{1} \bmod \mathscr{I} . }
\end{aligned}
$$

The tableau of this system is $[J, \kappa]$. (Remark: Do not confuse the tableau of the differential system to solve for $m$-subsets with the $m$-tableau of the solution m-subsets!) This tableau is involutive with characters $s_{1}=$ $\cdots=s_{s / 2}=s$ and $s_{(1+s) / 2}=0$. Thus maximal $\mathrm{m}$-subsets contained in $G_{2, s+2}$ depend locally on $s$ functions of $s / 2$ variables for $s$ even and it is easy to see that for $s$ odd, maximal involutive m-subsets contained in $G_{2, s+2}$ depend locally on $s-1$ functions of $(s-1) / 2$ variables. (We have already seen that all such involutive $m$-subsets must have CauchyRiemann m-tableaux.) Shortly we will see another proof of this giving a global answer.

This example is important because it shows that it is possible to have many nonisomorphic $\mathrm{m}$-subsets having the same $\mathrm{m}$-tableau and that there exist locally inhomogeneous $\mathrm{m}$-subsets (something not true locally for faces of parallel calibrations).

Call an m-tableau built out of Dirac m-tableaux of Clifford type. For example

$$
A=\left(\pi_{1}, J \pi_{1}, \pi_{2}, J \pi_{2}, 0\right) \subset \mathbf{R}^{s} \times \mathbf{R}^{5 *}
$$

is built from two Cauchy-Riemann m-tableaux and the zero m-tableau. Call an m-tableau $A$ of Clifford $^{*}$ type if there exists a metric on $W$ such 
that the dual tableau $A^{*}$ is of Clifford type.

Theorem 3.1. I. The only $m$-subsets having Clifford type m-tableaux have either $\mathbf{C}$ - or $\mathbf{H}$-Clifford type m-tableaux.

II. The only Clifford m-subsets having $\mathbf{H}$-Clifford type m-tableaux are the associative and Cayley faces.

III. The only $m$-subsets having C-Clifford type $m$-tableaux are:

A. The complex Grassmannians.

B. $(R$. Bryant $)$ C-Dirac type $m$-subsets (so $n=2)$, which correspond to complex $\frac{s}{2}$-dimensional subvarieties of $G_{2, s+2} \cong Q_{s} \subset \mathbf{C P}^{s+1}$ satisfying a transversality condition (where $Q_{s}$ is the null quadric).

C. C-Clifford type m-subsets in codimension 2, which correspond to complex $\frac{n}{2}$-dimensional subvarieties of $G_{n, n+2} \cong Q_{n} \subset \mathbf{C P}^{n+1}$ satisfying a transversality condition (where $Q_{n}$ is the null quadric).

D. $m$-subsets whose m-tableaux are of the type in $\mathrm{B}$ or $\mathrm{C}$ augmented by zeros, which are plentiful (i.e., they are 'parametrized' by arbitrary functions). (For an explanation of augmentation by zeros see $\S 5$.)

Theorem 3.1 ${ }^{*}$. I. The only m-subsets having Clifford ${ }^{*}$ type m-tableaux have m-tableaux dual to either $\mathbf{C}$ - or $\mathbf{H}$-Clifford type m-tableaux.

II. The only Clifford ${ }^{*}$ m-subsets whose duals have H-Clifford type $m$ tableaux are the coassociative and Cayley faces.

III. Same as 3.1.III by self-duality of the Cauchy-Riemann equations (i.e., if $A$ is a C-Clifford type $m$-tableau then so is $A^{*}$ ).

Corollary 3.2. The only $m$-subsets of codimension $\max (n, s)$ are the associative, coassociative, and Cayley faces and $G\left(\mathbf{C}, \mathbf{C}^{m+1}\right)$. (Codimension less than $\max (n, s)$ is impossible by Lemma I.2.4.)

Remark. If we restrict to studying faces of calibrations then the analogous statements to 3.1 and $3.1^{*}$ can be proven just by checking that the Dirac m-tableau generate the full orthogonal algebra under Lie bracket except for the exceptions above. In other words, one need only show that there does not exist $G, H \subset \mathrm{SO}(n)$ such that $A=T_{[e]} G / H$.

Proofs. With the exception of III, the proofs go as follows: the differential system for Dirac m-subsets will have a unique integral element at the first stage. In other words, the 1 -jet of a solution will uniquely determine the 2-jet. Then the prolonged system will either be Frobenius or have unabsorbable torsion. In other words, there will either be a unique solution or incompatible conditions will be placed on the 3-jet of a solution. The Frobenius systems will be the structure equations for a Lie algebra, in fact the two Frobenius cases are the structure equations for the algebras $\mathfrak{g}_{2}$ and $\mathfrak{s p i n}(7)$ for the associative and Cayley faces respectively. 
Case 1. Dirac m-subsets with $\mathbf{H}$-Dirac type $m$-tableaux in $G_{3,3+s}(s$ must be divisible by 4$)$. Write the m-tableau as $\left(\pi_{1}, \pi_{2}, J_{1} \pi_{1}+J_{2} \pi_{2}\right)$, where $J_{i}^{2}=-$ Id and $J_{1} J_{2}=-J_{2} J_{1}$. Take $\mathscr{I}=\left\{\theta=\psi_{3}-J_{1} \psi_{1}-J_{2} \psi_{2}\right\}$ and $\Omega=\psi_{1}^{1} \wedge \cdots \wedge \psi_{1}^{s} \wedge \psi_{2}^{1} \wedge \cdots \wedge \psi_{2}^{s}$. Then

$$
\begin{aligned}
d \theta= & -\kappa \wedge \psi_{3}-\psi \wedge \tau_{3}+J_{1}\left(\kappa \wedge \psi_{1}+\psi \wedge \tau_{1}\right)+J_{2}\left(\kappa \wedge \psi_{2}+\psi \wedge \tau_{2}\right) \\
\equiv & \left(\left[J_{1}, \kappa\right]+\tau_{1}^{2} J_{2}+\tau_{2}^{3} J_{1} J_{2}\right) \wedge \psi_{1} \\
& +\left(\left[J_{2}, \kappa\right]+\tau_{2}^{1} J_{1}+\tau_{3}^{1} J_{1} J_{2}\right) \wedge \psi_{2} \bmod \mathscr{I}
\end{aligned}
$$

This system has a unique integral element; i.e., to have $d \theta=0$ we must have $\left[J_{1}, \kappa\right]+\tau_{1}^{2} J_{2}+\tau_{2}^{3} J_{1} J_{2}$ and $\left[J_{2}, \kappa\right]+\tau_{2}^{1} J_{1}+\tau_{3}^{1} J_{1} J_{2}$ both identically zero. Thus we prolong (i.e., add these forms to the system), with the prolonged system defined on the same space. Set

$$
\mathscr{I}_{+}=\left\{\theta, \Theta_{1}:=\left[J_{1}, \kappa\right]+\tau_{1}^{2} J_{2}+\tau_{2}^{3} J_{1} J_{2}, \Theta_{2}:=\left[J_{2}, \kappa\right]+\tau_{2}^{1} J_{1}+\tau_{3}^{1} J_{1} J_{2}\right\} .
$$

Write $\kappa=\kappa^{\prime}+k_{1} J_{1}+k_{2} J_{2}+k_{3} J_{1} J_{2}$, where $\kappa^{\prime}$ is both $J_{1}$ and $J_{2}$ linear, and the $k_{j}$ are (scalar valued) one-forms.

$\Theta_{1}=0$ says

$$
2 k_{2}=\tau_{3}^{2}, \quad 2 k_{3}=\tau_{1}^{2},
$$

and $\Theta_{2}=0$ adds

$$
2 k_{1}=\tau_{3}^{1} \text {. }
$$

Notice that $\kappa^{\prime}$ does not appear in the tableau so it is metric dual to Cauchy characteristics. More precisely, the vector fields coming from curves of quaternionic linear transformations within $\mathrm{Gl}(s, \mathbf{R})$ are Cauchy characteristics and if we wanted to, we could set up the differential system on $\left(\mathrm{Gl}(s, \mathbf{R}) / \mathrm{Gl}\left(\frac{s}{4}, \mathbf{H}\right)\right) \times \mathrm{SO}(s+3)$, 'quotienting out' by the Cauchy characteristics.

In fact the only relevant nonzero vector fields on $\mathrm{Gl}(s, \mathbf{R})$ must be on $\mathrm{O}(s)$. We could work on $\mathrm{O}(s) \times \mathrm{SO}(s+3)$, but the $\mathrm{O}(s)$ action is redundant except for a $Z_{2}$ action, which we ignore since we are interested in connected solutions. So we now work on $\mathrm{SO}(s+3)$. We are fortunate because the basis of the ideal we were using for the system on $\operatorname{Gl}(s, \mathbf{R}) \times$ $\mathrm{SO}(s+3)$ is basic to the projection to $\mathrm{SO}(s+3)$ so we may work with the same forms. Continuing with the equations of the prolonged system, now that $\kappa \in \mathfrak{s o}(s)$ and $B \equiv \mathrm{Id}$, we have 


$$
\begin{aligned}
& d \boldsymbol{\Theta}_{1}=\left[J_{1},-\kappa \wedge \kappa+\psi \wedge^{t} \psi\right]+\left(-\tau_{3}^{2} \tau_{1}^{3}+\psi_{2}^{j} \wedge \psi_{1}^{j}\right) J_{2} \\
& +\left(-\tau_{1}^{3} \tau_{2}^{1}+\psi_{3}^{j} \wedge \psi_{2}^{j}\right) J_{3}
\end{aligned}
$$

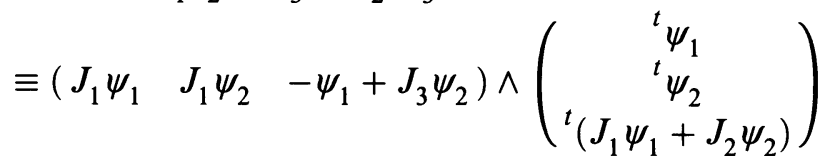

$$
\begin{aligned}
& +\left(\begin{array}{lll}
\psi_{1} & \psi_{2} & \left.J_{1} \psi_{1}+J_{2} \psi_{2}\right) \wedge \\
{ }^{t}\left(-\psi_{1}+J_{3} \psi_{2}\right)
\end{array}\right) \\
& +\sum_{j}\left\{\left(\psi_{2}^{j} \wedge \psi_{1}^{j}\right) J_{2}+\left(\left(J_{1} \psi_{1}\right)^{j} \wedge \psi_{2}^{j}\right) J_{3}+\left(\left(J_{2} \psi_{2}\right)^{j} \wedge \psi_{2}^{j}\right) J_{3}\right\} \bmod \mathscr{F}_{+} .
\end{aligned}
$$

The equations for the derivative of $\Theta_{2}$ are similar and thus the prolonged tableau is zero. If $s>4$ there is torsion and thus no solutions. However in the case $s=4$, the right-hand side of (3.5) is identically zero (as is the corresponding expression for $d \Theta_{2}$ ). This gives us a Frobenius system, in fact, the equations for a copy of $\mathfrak{g}_{2} \subset \mathfrak{s o}(7)$, i.e.,

$$
\begin{aligned}
\mathfrak{g}_{2} & =\left(\begin{array}{cc}
\tau & -{ }^{t} \psi \\
\psi & \kappa^{\prime}+\frac{1}{2}\left(\tau_{3}^{1} J_{1}+\tau_{3}^{2} J_{2}+\tau_{1}^{2} J_{1} J_{2}\right)
\end{array}\right) \\
& =\left(\begin{array}{cc}
\rho\left(\mathfrak{s p}(1)_{1}\right) & -{ }^{t} A \\
A & \mathfrak{s p}(1)_{2}+\mathfrak{s p}(1)_{1}
\end{array}\right),
\end{aligned}
$$

where $\rho\left(\mathfrak{s p}(1)_{1}\right)$ is a representation isomorphic to the adjoint.

Thus any $\mathrm{m}$-subset in $G_{3,7}$ with $\mathrm{m}$-tableau isomorphic to the associative tableau is an associative face. In fact, one can say more:

Corollary 3.3. Any smooth eight-dimensional $m$-subset of $G_{3,7}$ is (an open subset of ) an associative face.

Proof. By the classification of $\mathrm{m}$-tableaux in Part $\mathrm{I}$, any eight-dimensional $\mathrm{m}$-tableau of $\mathbf{R}^{4} \otimes \mathbf{R}^{3 *}$ is the associative $\mathrm{m}$-tableau. q.e.d.

Case 2. Dirac m-tableaux in $G_{4, s+4}$ (again, $s$ must be divisible by 4 ). Here we may write our m-tableau as $\left(\pi_{1}, \pi_{2}, \pi_{3}, J_{1} \pi_{1}+J_{2} \pi_{2}+J_{3} \pi_{3}\right)$, where $J_{i}^{2}=-$ Id and $J_{i} J_{j}=-J_{j} J_{i}$. Take

$$
\mathscr{I}=\left\{\theta:=\psi_{4}-J_{1} \psi_{1}-J_{2} \psi_{2}-J_{3} \psi_{3}\right\}
$$

and

$$
\Omega=\psi_{1}^{1} \wedge \cdots \wedge \psi_{1}^{s} \wedge \psi_{2}^{1} \wedge \cdots \wedge \psi_{2}^{s} \wedge \cdots \wedge \psi_{3}^{1} \wedge \cdots \wedge \psi_{3}^{s}
$$


Taking derivatives, we have

$$
\begin{aligned}
d \theta= & -\kappa \wedge \psi_{4}-\psi \wedge \tau_{4}+J_{1}\left(\kappa \wedge \psi_{1}+\psi \wedge \tau_{1}\right) \\
& +J_{2}\left(\kappa \wedge \psi_{2}+\psi \wedge \tau_{2}\right)+J_{3}\left(\kappa \wedge \psi_{3}+\psi \wedge \tau_{3}\right) \\
\equiv & \left(\left[J_{1}, \kappa\right]+\tau_{2}^{1} J_{2}+\tau_{4}^{2} J_{1} J_{2}+\tau_{3}^{1} J_{3}+\tau_{3}^{4} J_{3} J_{1}\right) \wedge \psi_{1} \\
& +\left(\left[J_{2}, \kappa\right]+\tau_{1}^{2} J_{1}+\tau_{1}^{4} J_{1} J_{2}+\tau_{3}^{2} J_{3}+\tau_{3}^{4} J_{3} J_{2}\right) \wedge \psi_{2} \\
& +\left(\left[J_{3}, \kappa\right]+\tau_{1}^{3} J_{1}+\tau_{1}^{4} J_{1} J_{3}+\tau_{2}^{3} J_{2}+\tau_{2}^{4} J_{2} J_{3}\right) \wedge \psi_{3} \bmod \mathscr{J} .
\end{aligned}
$$

This system has a unique integral element, so we prolong, adding the forms in the tableau to the ideal. Writing $\kappa=\kappa^{\prime}+k_{1} J_{1}+k_{2} J_{2}+k_{3} J_{3}$, as in (3.3) and (3.4), we add the following forms to the ideal:

$$
2 k_{2}-\left(\tau_{2}^{4}+\tau_{1}^{3}\right), \quad 2 k_{3}-\left(\tau_{2}^{1}+\tau_{3}^{4}\right), \quad 2 k_{1}-\left(\tau_{1}^{4}+\tau_{3}^{2}\right)
$$

As with the case above, we may quotient out the $\mathrm{GL}(s)$ action and define the system on $\mathrm{SO}(4+s)$. Again, the tableau of the prolonged system is zero. If $s>4$ we get torsion and therefore no solutions. Even in the case $s=4$, we get torsion unless $J_{1} J_{2} J_{3}= \pm \mathrm{Id}$, i.e., unless we have an H-module structure. In this case the system is a Frobenius system, in fact the equations for a copy of $\mathfrak{s p i n}(7) \subset \mathfrak{s o}(8)$, i.e.,

$$
\mathfrak{s p i n}(7)=\left(\begin{array}{cc}
\mathfrak{s p}(1)_{1}+\rho\left(\mathfrak{s p}(1)_{2}\right) & -{ }^{t} A \\
A & \mathfrak{s p}(1)_{3}+\tilde{\rho}\left(\mathfrak{s p}(1)_{2}\right)
\end{array}\right),
$$

where the columns of $A$ satisfy $J_{1} \pi_{1}+J_{2} \pi_{2}+J_{3} \pi_{3}-\pi_{4}=0$, and $\rho$ and $\tilde{\rho}$ are both isomorphic to the standard representations, but given in terms of different, nonstandard bases.

Thus any $\mathrm{m}$-subset in $G_{4,8}$ with m-tableau isomorphic to the Cayley m-tableau is a Cayley face, and again by the classification in Part I we have:

Corollary 3.4. Any smooth twelve-dimensional $m$-subset of $G_{4,8}$ is (an open subset of ) a Cayley face.

Any larger Dirac m-tableau must contain one of the $s>4$ cases and the same equations that gave unabsorbable torsion will reappear, so they will not have any solutions. The reader may check that horizontally stacked H-Dirac type m-tableaux have no corresponding solution m-subsets (the computation is similar to, but easier than, the proof of Case 2), and finally Theorems 5.1 and 5.2 will prove the extension of I and II from Dirac to Clifford m-tableaux.

We now prove the $G_{n, n+3}$ case of II $^{*}$ : Here the m-tableau is the transpose of (3.2). Write it as 


$$
\left(\begin{array}{c}
\pi^{1} \\
\pi^{2} \\
\pi^{1} J_{1}+\pi^{2} J_{2}
\end{array}\right),
$$

where $\pi^{j}=\left(\begin{array}{lll}\pi_{1}^{j} & \cdots & \pi_{n}^{j}\end{array}\right)$ and we have changed the signs on the complex structures for notational simplicity. Here

$$
\mathscr{I}=\left\{\theta:=\psi^{3}-\psi^{1} J_{1}-\psi^{2} J_{2}\right\}
$$

and

$$
\Omega=\psi_{1}^{1} \wedge \cdots \wedge \psi_{n}^{1} \wedge \psi_{1}^{2} \wedge \cdots \wedge \psi_{n}^{2} .
$$

Taking derivatives, we have

$$
\begin{gathered}
d \theta=-\kappa^{3} \wedge \psi-\psi^{3} \wedge \tau+\left(\kappa^{1} \wedge \psi+\psi^{1} \wedge \tau\right) J_{1}+\left(\kappa^{2} \wedge \psi+\psi^{2} \wedge \tau\right) J_{2} \\
\equiv \psi^{1} \wedge\left\{\left[\tau, J_{1}\right]+\left(\kappa_{1}^{3}+\kappa_{3}^{1}\right) \operatorname{Id}+\left(\kappa_{3}^{3}-\kappa_{1}^{1}\right) J_{1}+\kappa_{2}^{1} J_{2}+\kappa_{2}^{3} J_{1} J_{2}\right\} \\
\quad+\psi^{2} \wedge\left\{\left[\tau, J_{2}\right]+\left(\kappa_{2}^{3}+\kappa_{3}^{2}\right) \operatorname{Id}\right. \\
\left.\quad+\left(\kappa_{3}^{3}-\kappa_{2}^{2}\right) J_{2}-\kappa_{1}^{2} J_{1}-\kappa_{1}^{3} J_{1} J_{2}\right\} \bmod \mathscr{I} .
\end{gathered}
$$

This system has a unique integral element, so we prolong. Write $\tau=$ $\tau^{\prime}+t_{1} J_{1}+t_{2} J_{2}+t_{3} J_{1} J_{2}$ as we did with $\kappa$ in Case 1 . With this notation,

$$
\begin{aligned}
\mathscr{I}_{+}=\left\{\theta, \kappa_{3}^{1}+\kappa_{1}^{3}, \kappa_{3}^{2}+\kappa_{2}^{3}, \kappa_{3}^{3}-\kappa_{1}^{1},\right. & \kappa_{3}^{3}-\kappa_{2}^{2}, \kappa_{2}^{1}-2 t_{3}, \\
& \left.\kappa_{2}^{3}-2 t_{2}, \kappa_{1}^{2}+2 t_{3},-\kappa_{1}^{3}+2 t_{1}\right\} .
\end{aligned}
$$

Following the associative computation (and recalling that we are free to define the system on $\operatorname{SL}(3, \mathbf{R}) \times \mathrm{SO}(n+3))$ we see that $\mathscr{I}_{+}$has unabsorbable torsion unless $n=4$, in which case it is a Frobenius system for copies of $\mathfrak{g}_{2} \subset \mathfrak{s o}(7)$. By the classification of Part I, the only eight-dimensional m-tableau in $G_{4,7}$ is the coassociative m-tableau, so we have:

Corollary 3.5. Any smooth eight-dimensional $m$-subset of $G_{4,7}$ is (an open subset of ) a coassociative face.

The proofs for Dirac ${ }^{*}$ m-subsets in $G_{n, n+4}$ and larger Grassmannians are similar to the above. We leave the details to the reader. Theorems 5.1 and 5.2 will complete the proof of Theorem $3.1^{*}$, parts I and II.

We now prove part III. We have already seen C-Dirac type m-tableaux have, at least locally, many corresponding m-subsets and now we show the global result stated in the theorem. It may be thought of as a generalization in one direction of the classical theorem that $M^{2} \stackrel{f}{\longrightarrow} \mathbf{R}^{s+2}$ is minimal if and only if the Gauss map $M^{2} \stackrel{\gamma_{f}}{\longrightarrow} G_{2, s+2} \cong Q_{s} \subset \mathbf{C P}^{s+1}$ is holomorphic. 
Proposition 3.6 (R. Bryant). If $\gamma_{f}\left(M^{2}\right)$ is a complex submanifold of $G_{2, s+2} \cong Q_{s}$ whose tangent space contains no decomposable vectors, then $f(M)$ is minimal. More generally, any complex submanifold $\Sigma \subset G_{2, s+2} \cong$ $Q_{s}$, such that $T_{E} \Sigma$ contains no decomposable vectors for all $E \in \Sigma$, is an m-subset.

Proof. Let $e^{1}, e^{2}$ be an orthonormal basis of $E^{*}$ and $v, w \in E^{\perp}$. The nondecomposability requirement says that if $v \otimes e^{1}+w \otimes e^{2} \in T_{E} \Sigma$, then $v, w$ must be linearly independent. Write the complex structure $J$ as

$$
J\left(v \otimes e^{1}+w \otimes e^{2}\right)=-w \otimes e^{1}+v \otimes e^{2} .
$$

Then we may choose a basis of $E^{\perp}$ such that $T_{E} \Sigma$ is spanned by

$$
\begin{aligned}
& \left\{v_{1} \otimes e^{1}+v_{2} \otimes e^{2}, \cdots, v_{t-1} \otimes e^{1}+v_{t} \otimes e^{2},\right. \\
& \left.-v_{2} \otimes e^{1}+v_{1} \otimes e^{2}, \cdots,-v_{t} \otimes e^{1}+v_{t-1} \otimes e^{2}\right\},
\end{aligned}
$$

where $\Sigma$ is $t$ real dimensional, i.e.,

$$
T_{E} \Sigma \cong\left(\begin{array}{cc}
v_{1} & v_{2} \\
-v_{2} & v_{1} \\
\vdots & \vdots \\
v_{t-1} & v_{t} \\
-v_{t} & v_{t-1} \\
0 & 0 \\
\vdots & \vdots \\
0 & 0
\end{array}\right),
$$

which is clearly an m-tableau, in fact a Cauchy-Riemann m-tableau, so $\Sigma$ is an $\mathrm{m}$-subset. q.e.d.

Since the generic linear subspace of $E^{\perp} \otimes E^{*}$ of complex dimension $\left[\frac{s}{2}\right]$ will satisfy this transversality condition, we have:

Corollary 3.7 (R. Bryant). The generic complex submanifold of $G_{2, s+2}$ of complex dimension $\left[\frac{s}{2}\right]$ is an m-subset.

Remark. Proposition 3.6 has the unfortunate consequence of showing that, in general, $\mathrm{m}$-subsets do not have global properties. Micallef [11] has shown that any complete, oriented, nonholomorphic, parabolic minimal surface in $\mathbf{R}^{4}$ is unstable. The image of the Gauss map of a minimal surface in $G_{2,4}$ is a complex submanifold. In general the image may contain decomposable tangent spaces but these should occur at isolated points, and the incomplete submanifold obtained by removing the points 
would still be unstable, even though its Gauss map takes images in an msubset. For an example of an unstable $\Sigma$-manifold, see Osserman [14, p. 42].

The C-Dirac m-subsets give a large class of $\mathrm{m}$-subsets having a given m-tableau, but only for minimal surfaces, which are already fairly well understood. However $G_{2, n+2} \cong G_{n, n+2}$ and the same proof (transposed) gives:

Proposition 3.8. Complex submanifolds $\Sigma \subset G_{n, n+2}$ whose tangent spaces contain no decomposable vectors are $m$-subsets.

Corollary 3.9. The generic complex submanifold of $G_{n, n+2}$ of complex dimension $\left[\frac{n}{2}\right]$ is an m-subset.

Some homogeneous examples of this type of m-subset which are not contained in any complex Grassmannian are given by representations of $\mathrm{SU}(n+1)$.

Theorem 3.10. Let $V^{N}$ denote any irreducible real $\mathrm{SU}(n+1)$ module other than the adjoint. Then the orbits of certain codimension-2 planes are $m$-subsets (which ones will be made clear in the proof).

Remark. We deal with the real representations here because the msubsets resulting from complex representations will all be contained in some standard complex Grassmanian.

Proof. Choose a basis of $V$ such that a maximal torus of $\mathfrak{s u}(n+1)$ is on diagonal $2 \times 2$ blocks and choose a real eigenbasis for the weight zero space. With this basis, every basis vector will either map to zero or remain in a 2-plane under the action of the torus. Order the basis such that the highest weight $\lambda$ acis on the last two vectors.

Claim. The orbit of the codimension two plane orthogonal to the plane of highest weight is an m-subset. The tangent space of this orbit in $G_{N-2, N}$ is isomorphic to the lower left-hand $2 \times(N-2)$ block of the image of $\mathfrak{s u}(n+1)$ in $\operatorname{end}(V)(N=\operatorname{dim}(V))$.

Since the weight $\lambda$ can only act on its nearest neighbors, the block will be mostly zero. There will be $n$ nonzero $2 \times 2$ subblocks corresponding to the neighbors of $\lambda$ on the outer 'shell' of weights of multiplicity one, and two other nonzero subblocks coming from the neighbor to $\lambda$ on the next shell of weights of multiplicity two. The tableau is $2 n$-dimensional consisting of zeros and $2 \times 2$ blocks of the form

$$
\left(\begin{array}{cc}
c_{j} a_{j} & -c_{j} b_{j} \\
c_{j} b_{j} & c_{j} a_{j}
\end{array}\right), \quad 1 \leq j \leq n
$$

where the $c_{j}$ 's are constants and the $a_{j}$ 's and $b_{j}$ 's are free, and one block 
of the form

$$
\left(\begin{array}{cc}
c_{0} a_{1} & -c_{0} b_{1} \\
c_{0} b_{1} & c_{0} a_{1}
\end{array}\right),
$$

where $c_{0}$ is a constant and $a_{1}$ and $b_{1}$ are associated to the weight $\lambda-$ $(1, \cdots, 1)$ which occurs with multiplicity two. This is clearly an involutive m-tableau (under a change of basis it is a standard Cauchy-Riemann m-tableau with zeros).

Remark. The $\Sigma$-manifolds given by Theorem 3.10 are all austere (see [8, p. 102]). By a result of Bryant in [3], they must have singularities. As with all austere submanifolds, they may be used to construct new examples of special Lagrangian submanifolds.

Remark. The $\Sigma$-manifolds associated to these m-subsets are ruled by $(N-2 n-2)$-planes and have the following geometric description: Let $\mathscr{L}$ denote the subspace of the Grassmann bundle $G_{N-2 n-2, N} \times \mathbf{R}^{N}$ given by the $\rho(\mathrm{SU}(n+1)) \times \mathbf{R}^{N}$ orbit of a special $(N-2 n-2)$-plane, where $\rho$ denotes the map $\mathrm{SU}(n+1) \rightarrow \mathrm{SO}(N)$. $\mathscr{L}$ is a complex manifold which arises naturally as follows: We solve for the $\Sigma$-manifolds by solving for their lifts to the $\rho(\operatorname{SU}(n+1))$-coframe bundle $\mathscr{F}$, and $\mathscr{L}$ is $\mathscr{F}$ quotiented out by the Cauchy characteristics of the system to solve for lifts of $\Sigma$-manifolds.

A $\Sigma$-manifold $M$ is obtained by taking complex $n$-folds in $\mathscr{L}$ that solve the differential system induced from $\mathscr{F}$. This gives a $2 n$-parameter family of special $(N-2 n-2)$-planes whose union fills out $M$. (One lifts the $n$-fold in $\mathscr{L}$ to $\mathscr{F}$ and pushes it back down to $\mathbf{R}^{N}$.)

The $\Sigma$-manifolds corresponding to the simplest solutions of the differential system on $\mathscr{L}$ are homogeneous cones, in fact the $\mathrm{SU}(n+1)$ orbit of a special $(N-2 n-2)$-plane in $\mathbf{R}^{N}$. (In coordinates the general solutions are functions graphed over a complex $n$-plane and the simplest solution corresponds to the complex $n$-plane itself, i.e., the case where all the functions are identically zero.) In other words, we have the following double fibration picture:

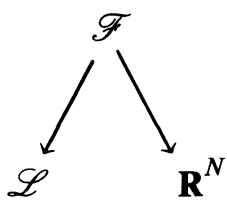

where $\Sigma$-manifolds are obtained by lifting certain complex $n$-folds in $\mathscr{L}$ to $\mathscr{F}$ and then projecting them down to $\mathbf{R}^{N}$. 
Remark. In [9] an explicit Weierstrass-type representation formula is given for the 3-dimensional $\Sigma$-manifolds in $\mathbf{R}^{5}$. We show in the appendix that these 3 -folds are locally calibrated.

Remark. m-subsets consisting of orbits of real representations of SU(2) are studied in all codimensions in [9]. These give examples of $\mathrm{m}$-subsets having the $2 \times 2$ Cauchy-Riemann $m$-tableau augmented by zeros as their m-tableau.

We now prove the rigidity part of III, i.e., that for $s, n>2$, C-Clifford type m-tableaux have unique solution m-subsets up to isomorphism, corresponding to complex Grassmannians.

Proof. In $G_{2 p+q, 2 p+q+s}$ ( $s$ must be even), write

$$
A=\left(\pi_{1}, \cdots, \pi_{p}, J \pi_{1}, \cdots, J \pi_{p}, 0, \cdots, 0\right) .
$$

Let $\theta_{j}=\psi_{p+j}-J \psi_{j}, \theta_{r}=\psi_{r}, 1 \leq j \leq p, 2 p+1 \leq r \leq n=2 p+q$. Take $\mathscr{F}=\left\{\theta_{j}, \theta_{r}\right\}$, and $\Omega=\psi_{1}^{1} \wedge \cdots \wedge \psi_{1}^{s} \wedge \cdots \wedge \psi_{p}^{1} \wedge \cdots \wedge \psi_{p}^{s}$. Taking derivatives, we have

$$
\begin{aligned}
d \theta_{j}= & -\kappa \wedge \psi_{p+j}-\psi \wedge \tau_{p+j}+J\left(\kappa \wedge \psi_{j}-\psi \wedge \tau_{j}\right) \\
\equiv & -\kappa \wedge J \psi_{p}-\left(\psi_{1}, \cdots, \psi_{j}, \cdots, J \psi_{1}, \cdots, J \psi_{p}, 0, \cdots, 0\right) \wedge \tau_{p+j} \\
& +J\left(\kappa \wedge \psi_{j}-\left(\psi_{1}, \cdots, \psi_{p}, \cdots, J \psi_{1}, \cdots,\right.\right. \\
& \left.\left.\quad J \psi_{p}, 0, \cdots, 0\right) \wedge \tau_{j}\right) \bmod \mathscr{I} \\
\equiv & {[J, \kappa] \wedge \psi_{j}+\Gamma_{j}^{k} \wedge \psi_{k} \bmod \mathscr{I}, }
\end{aligned}
$$

where $\Gamma_{j}^{k}=\left(\tau_{p+j}^{k}+\tau_{j}^{k+p}\right) \mathbf{I d}+\left(\tau_{p+j}^{k+p}-\tau_{j}^{k}\right) J$ (notice that $\left.\Gamma_{j}^{k}=-\Gamma_{k}^{j}\right)$, and

$$
d \theta_{r}=-\kappa \wedge \psi_{r}-\psi \wedge \tau_{r} \equiv \Gamma_{j}^{r} \wedge \psi_{j} \bmod \mathscr{I},
$$

where $\Gamma_{j}^{r}=-\tau_{r}^{j} \operatorname{Id}+\tau_{r}^{p+j} J$.

We may write the tableau of this system in block form:

$$
\left(\begin{array}{ccccccc}
{[J, \kappa]} & \Gamma_{2}^{1} & \cdots & \Gamma_{p}^{1} & -{ }^{t} \Gamma_{1}^{p+1} & \cdots & -{ }^{t} \Gamma_{1}^{p+q} \\
\Gamma_{1}^{2} & {[J, \kappa]} & & \vdots & \vdots & \ddots & \vdots \\
\vdots & & \ddots & \Gamma_{p}^{p-1} & & & \\
\Gamma_{1}^{p} & \ldots & \Gamma_{p-1}^{p} & {[J, \kappa]} & -{ }^{t} \Gamma_{1}^{p+1} & \cdots & -{ }^{t} \Gamma_{1}^{p+q} \\
\Gamma_{1}^{p+1} & \cdots & & \Gamma_{p}^{p+1} & 0 & \cdots & 0 \\
\vdots & & & \vdots & \vdots & \ddots & \vdots \\
\Gamma_{1}^{p+q} & \ldots & & \Gamma_{p}^{p+q} & 0 & \cdots & 0
\end{array}\right) .
$$

It is easy to see this system has a unique integral element. 
Notice that $\Gamma_{j}^{r}=0$ implies $\tau_{r}^{j}, \tau_{r}^{p+j}=0(p>1)$ which reduces $\tau$ to lie in $\mathfrak{s o}(2 p)$ and shows that, at least in this case, adding zeros to an m-tableau will not provide a more general class of m-subsets. (A general discussion on adding zeros is given in $\S 5$.) To avoid cumbersome notation we continue the computation for the m-tableau $\left(\pi_{1}, \pi_{2}, J \pi_{1}, J \pi_{2}\right)$ for m-subsets in $G_{4, s+4}$ and the general case will be clear from this one.

In this case the tableau is

$$
\left(\begin{array}{cc}
{[J, \kappa]} & -t_{1} \mathrm{Id}+t_{2} J \\
t_{1} \mathrm{Id}-t_{2} J & {[J, \kappa]}
\end{array}\right),
$$

where $t_{1}=\tau_{4}^{1}+\tau_{2}^{3}$ and $t_{2}=\tau_{3}^{4}-\tau_{1}^{2}$.

Notice that $t_{1}$ and $t_{2}$ occur on every row. To see there is a unique integral element in this case, we may choose bases such that a zero occurs in every column as long as $s>2$ (e.g., take bases such that $J=\left(\begin{array}{cc}0 & -I \\ I & 0\end{array}\right)$. Thus on an integral element we must have $t_{1}=t_{2}=0$. What remains of the tableau is

$$
\left(\begin{array}{cc}
{[J, \kappa]} & 0 \\
0 & {[J, \kappa]}
\end{array}\right)
$$

which implies $[J, \kappa]=0$ on an integral element.

The prolonged system is defined on the same space with the same independence condition and the ideal $\mathscr{I}_{+}=\left\{\theta_{1}, \theta_{2},[J, \kappa], t_{1}, t_{2}\right\}$. Now

$$
d t_{1}=-\tau_{2}^{1} \wedge \tau_{4}^{2}-\tau_{3}^{1} \wedge \tau_{4}^{3}+{ }^{t} \psi_{1} S \wedge \psi_{4}-\tau_{1}^{3} \wedge \tau_{2}^{1}-\tau_{4}^{3} \wedge \tau_{2}^{4}+{ }^{t} \psi_{3} S \wedge \psi_{2},
$$

where $S={ }^{t} B B$, and

$$
\begin{aligned}
d t_{1} & \equiv{ }^{t} \psi_{1} S \wedge J \psi_{2}+{ }^{t}\left(J \psi_{1}\right) S \wedge \psi_{2} \bmod \mathscr{I}_{+} \\
& \equiv{ }^{t} \psi_{1}[S, J] \wedge \psi_{2} \bmod \mathscr{I}_{+} .
\end{aligned}
$$

Since $\psi_{1}$ and $\psi_{2}$ have independent entries we must restrict the system to the submanifold of $\mathrm{Gl}(s) \times \mathrm{SO}(4+s)$ on which $[S, J]=0$ to get rid of the torsion. (The equations for $d t_{2}$ give the same thing.)

The next thing to do is to check if this restriction implies any new relations:

$$
\begin{aligned}
d[S, J] & =\left[\left(d^{t} B\right) B+{ }^{t} B(d B), J\right] \\
& =\left[{ }^{t}(B \kappa-\tilde{\delta} B) B+{ }^{t} B(B \kappa-\tilde{\delta} B), J\right] \\
& =\left[{ }^{t} \kappa S+S \kappa, J\right]=\left[{ }^{t} \kappa, J\right] S+S[\kappa, J],
\end{aligned}
$$

which already was in $\mathscr{I}_{+}$(as $\left.\left[{ }^{t} \kappa, J\right]=-{ }^{t}[\kappa, J]\right)$.

The reader may now check that $d[\kappa, J] \equiv 0 \bmod \mathscr{J}_{+}$restricted to the submanifold $[S, J]=0$, so $\mathscr{I}_{+}$restricted to the submanifold $[S, J]=0$ is Frobenius. 
Claim. The solutions correspond to complex Grassmannians, $G\left(\mathbf{C}^{2}, \mathbf{C}^{2+s / 2}\right) \subset G_{4, s+4}$.

Proof. Without loss of generality we may 'quotient out' by some of the Cauchy characteristics and redefine the system on $W \times \operatorname{SO}(5)$, where

$$
W=\left\{\left.B \in \mathrm{GL}(s, \mathbf{R})\right|^{t} B=B,\left[B^{2}, J\right]=0, \operatorname{det}(B)=1\right\} .
$$

Claim. Any $B \in W$ may be written $B=A P B_{0}{ }^{t} A$, where

$$
A \in U_{J}(s / 2):=\{g \in \mathrm{SO}(s) \mid[g, J]=0\},
$$

$$
\begin{aligned}
& B_{0}=\left(\begin{array}{cccccc}
\lambda_{1} & & & & & \\
& \ddots & & & & \\
& & \lambda_{s / 2} & & & \\
& & & \lambda_{1} & & \\
& & & & \ddots & \\
& & & & & \lambda_{s / 2}
\end{array}\right), \quad \lambda_{i}>0, \lambda_{1} \cdots \lambda_{s / 2}=1 \text {, } \\
& P=\left(\begin{array}{ccc}
\varepsilon_{1} & & \\
& \ddots & \\
& & \varepsilon_{s}
\end{array}\right), \quad \varepsilon_{j}= \pm 1 .
\end{aligned}
$$

This is true because $B^{2}$ must be positive definite hermitian and thus expressible as $B^{2}=A\left(B_{0}^{2}\right)^{t} A$ which has $2 s$ possible square roots accounted for by $P$.

On a solution we have

$$
\begin{aligned}
0=[\kappa, J]=\left[A \left(B_{0}^{-1} P A^{-1} d A P B_{0}+B_{0}^{-1}\right.\right. & d B_{0}-A^{-1} d A \\
& \left.\left.+B_{0}^{-1} P A^{-1} \tilde{\delta} A P B_{0}\right) A^{-1}, J\right],
\end{aligned}
$$

i.e.,

$$
\left[A^{-1} d A+A^{-1} \tilde{\delta} A, P J P\right]=0 .
$$

Notice that $B_{0}$ does not show up in the equations, so we can reduce further to define the system on the submanifold of $W \times \mathrm{SO}(s+4)$ on which $B_{0} \equiv \mathrm{Id}$. Now at this point $W$ is orthogonal, so we may as well restrict the system to $\mathrm{SO}(s+4)$. However, on $\mathrm{SO}(s+4)$ the system is easily recognized as the structure equations of a copy of $\mathrm{U}\left(\frac{s}{2}+2\right) \subset \mathrm{SO}(s+4)$. Thus the only solution to our system is a complex Grassmannian.

\section{4. $\mathbf{m}$-subsets with special Lagrangian $\mathbf{m}$-tableaux}

The only 'classical' m-tableau that remains to be analyzed is the special Lagrangian m-tableau. We have seen that the m-subsets with the special 
Lagrangian m-tableau in $G_{2,4}$ are far from rigid, in fact they may be specified in terms of holomorphic data. (In $G_{2,4}$ the Cauchy-Riemann mtableau is isomorphic to the special Lagrangian $\mathrm{m}$-tableau.) The situation in higher dimensions is quite different:

Theorem 4.1. Any smooth $m$-subset with $m$-tableau isomorphic to a special Lagrangian m-tableau is (an open subset of) a special Lagrangian face $(n>2)$.

Proof. Write the m-tableau as $A$ such that ${ }^{t} A=A$ and $\operatorname{trace}(A)=0$. Take

$$
\mathscr{I}=\left\{\theta:=\psi-{ }^{t} \psi, \theta_{0}:=\operatorname{trace}(\psi)\right\}
$$

and

$$
\Omega=\psi_{1}^{1} \wedge \cdots \wedge \psi_{1}^{n} \wedge \psi_{2}^{2} \wedge \cdots \wedge \psi_{2}^{n} \wedge \cdots \wedge \psi_{3}^{3} \wedge \cdots \wedge \psi_{n-1}^{n-1} .
$$

Taking derivatives, we have

$$
\begin{aligned}
d \theta & =-\kappa \wedge \psi-\psi \wedge \tau+{ }^{t}(\kappa \wedge \psi+\psi \wedge \tau) \\
& \equiv-\kappa \wedge \psi-\psi \wedge \tau-\psi \wedge{ }^{t} \kappa+\tau \wedge \psi \bmod \mathscr{F} .
\end{aligned}
$$

Let $\alpha=\tau-\kappa$; then we have

$$
\begin{gathered}
d \theta \equiv \alpha \wedge \psi+\psi \wedge^{t} \alpha \bmod \mathscr{I} \equiv \alpha \wedge \psi-{ }^{t}(\alpha \wedge \psi) \bmod \mathscr{I}, \\
d \theta_{0}=\operatorname{tr}(-\kappa \wedge \psi-\psi \wedge \tau) \equiv \operatorname{tr}(\alpha \wedge \psi) \bmod \mathscr{I} .
\end{gathered}
$$

Let $1 \leq i, j, k, l, m, p, q, t, w \leq s \quad(=n)$. On an integral element we must have

$$
\alpha^{i j} \equiv p_{k l}^{i j} \psi_{k l} \bmod \mathscr{I},
$$

where $p_{k l}^{i j}=p_{i j}^{k l}$ by the symmetry of $A^{(1)}, p_{k l}^{i j}=p_{l k}^{i j}$ because $\psi={ }^{t} \psi$, and no pair of indices $=(s s)$. Equations (4.1) and (4.2) say that $C:=\alpha \wedge \psi$ is such that $C={ }^{t} C$ and $\operatorname{tr}(C)=0$. Substituting for $\alpha, C_{m}^{i}=p_{k l}^{i j} \psi_{k l} \wedge \psi_{j m}$. $C$ symmetric says

$$
p_{k l}^{i j} \psi_{k l} \wedge \psi_{j m}=p_{w q}^{m t} \psi_{w q} \wedge \psi_{t i} .
$$

Say $j \neq i$ and $i \neq m$, then $p_{k l}^{i j}=0$ if $\{k, l\} \cap\{i\}=\varnothing$ because when $n>2$ there will be an $m$ such that the form $\psi_{k l} \wedge \psi_{j m}$ will appear only on the left-hand side of (4.3). Moreover, the term $p_{m q}^{m t} \psi_{m q} \wedge \psi_{t i}$ on the right-hand side must have a corresponding term on the left-hand side so (no sum over $i, m$ )

$$
\sum_{j, l} p_{i l}^{i j} \psi_{i l} \wedge \psi_{j m}=\sum_{t, q} p_{m q}^{m t} \psi_{m q} \wedge \psi_{t i}
$$


Picking out a single term (no sum)

$$
p_{i l}^{i j} \psi_{i l} \wedge \psi_{j m}=p_{m j}^{m l} \psi_{m j} \wedge \psi_{l i}=-p_{m j}^{m l} \psi_{i l} \wedge \psi_{j m}
$$

so $p_{i j}^{i l}=-p_{m j}^{m l}$ for $l, m, i, j$ disjoint, but using a cyclic permutation (again we need $n>2$ ) we have $p_{i j}^{i l}=0$ for $l, i, j$ disjoint. Now only the $p_{i j}^{i i}$ have not yet been shown to be zero, so $\alpha^{i j}=0$ for $i \neq j$. The trace condition

$$
\sum_{i} C_{i}^{i}=0
$$

gives $\alpha^{i j} \wedge \psi_{j i}=0$, where $\alpha^{i i}=p_{i l}^{i i} \psi_{i l}$ for $i<s$ and $\alpha^{s s}=-\sum_{i<s} \alpha^{i i}$. Expanding out (4.4) we see $p_{i l}^{i i}=0$, so $\alpha=0$ and thus there is a unique integral element.

The prolonged system is defined on the same manifold with ideal

$$
\mathscr{I}_{+}=\left\{\theta, \theta_{0}, \alpha\right\} \text {. }
$$

Notice that $\alpha=0 \Rightarrow \kappa=\tau \Rightarrow \kappa \in \mathfrak{s o}(s)$, so the system may actually be defined on $\mathrm{SO}(2 s)$ because the right $\mathrm{GL}(s)$ action only contributes a redundant orthogonal action to the system. In fact $\kappa=\tau$ together with the original conditions on $\psi$ specifies a Frobenius system for copies of $\mathrm{SU}(s) \subset \mathrm{SO}(2 s)$ and we may write

$$
\mathfrak{s u}(s)=\left(\begin{array}{cc}
\tau & -\psi \\
\psi & \tau
\end{array}\right),
$$

where $\tau \in \mathfrak{s o}(s / 2)$ and $\psi$ is traceless and symmetric.

\section{Adding zeros to an m-tableau}

Example. The m-tableau

$$
A=\left(\begin{array}{ccc}
a & -b & 0 \\
b & a & 0
\end{array}\right)
$$

has many solution m-subsets including two distinct homogeneous ones, $G\left(\mathbf{C}, \mathbf{C}^{2}\right) \subset G_{3,5}$, and a degenerate $\mathrm{SO}(3)$ orbit of a 3-plane in $\mathbf{R}^{5}$ (by Theorem 3.10). The reader may check in fact that the differential system to solve for m-subsets with m-tableau $A$ is involutive with tableau

$$
\left(\begin{array}{cc}
{[\kappa, J]} \\
\tau_{3}^{1} & -\tau_{3}^{2} \\
\tau_{3}^{2} & \tau_{3}^{1}
\end{array}\right)
$$


One gets a similar result for the m-tableau ${ }^{t} A$. This illustrates the claim in Theorem 3.1.III.D, and the reader can see the general situation from these two.

These examples might lead one to think that, in general, adding zeros to an m-tableau may enlarge the class of corresponding m-subsets. There are two ways to add zeros, 'horizontally' and 'vertically', i.e., given an mtableau $A$ for $G_{n, n+s}$ we consider the m-tableaux $(A, 0)$ for $G_{n+d, n+d+s}$ and $\left(\begin{array}{c}A \\ 0\end{array}\right)$ for $G_{n, n+w+s}$.

The minimal submanifolds produced by m-subsets with m-tableaux augmented by zeros are ruled by $d$-planes in the case of horizontal addition of zeros, and osculate to an $\mathbf{R}^{n+w} \subset \mathbf{R}^{n+w+s}$ to second order at each point in the case of vertical addition of zeros. (In the case that adding zeros does not produce new classes of $\mathrm{m}$-subsets, the minimal submanifolds produced by the $\mathrm{m}$-subsets having $\mathrm{m}$-tableaux with zeros are products of minimal $n$-folds and $d$-planes in the case of horizontal zeros and lie entirely in an $\mathbf{R}^{n+w} \subset \mathbf{R}^{n+w+s}$ in the case of vertical zeros.)

Theorem 5.1. If an involutive $m$-tableau $A$ has characters such that $s_{1}>2$, and $s_{2}=s_{1}$ or $s_{2}=\left(s_{1}-1\right)$, then 'adding vertical zeros' to $A$ will not enlarge its class of corresponding $m$-subsets. In particular $(s>2)$ adding vertical zeros to the Dirac or Dirac ${ }^{*} m$-tableaux (except for C-type) and special Lagrangian m-tableaux does not enlarge the class of solution m-subsets.

Proof. Say the system for m-subsets with $A$-type m-tableau is $\mathscr{I}=$ $\left\{\theta^{r}\right\}$, with independence condition $\Omega$. Then the system for m-subsets with $\left(\begin{array}{c}A \\ 0\end{array}\right)$-type m-tableau is $\mathscr{I}=\left\{\theta^{r}, \tilde{\psi}\right\}$, with independence condition $\Omega$, where

$$
\psi_{0}=\left(\begin{array}{c}
\psi \\
\tilde{\psi}
\end{array}\right), \quad \kappa_{0}=\left(\begin{array}{ll}
\kappa_{1} & \kappa_{3} \\
\kappa_{2} & \kappa_{4}
\end{array}\right), \quad \tau_{0}=\tau,
$$

where the zero subscript denotes the forms on the large Grassmannian. Taking the derivative of $\tilde{\psi}$ gives

$$
d \tilde{\psi}=-\kappa_{2} \wedge \psi-\kappa_{4} \wedge \tilde{\psi}-\tilde{\psi} \wedge \tau \equiv-\kappa_{2} \wedge \psi \bmod \mathscr{I}
$$

In particular, on an integral element,

$$
\begin{array}{ll}
(d \tilde{\psi})_{1}^{m} \equiv-\left(\kappa_{2}\right)_{a}^{m} \wedge(\psi)_{1}^{a} \bmod \mathscr{I} \Rightarrow\left(\kappa_{2}\right)_{a}^{m}=P_{a b}^{m} \psi_{1}^{b}, & P_{a b}^{m}=P_{b a}^{m} \\
(d \tilde{\psi})_{2}^{m} \equiv-\left(\kappa_{2}\right)_{a}^{m} \wedge(\psi)_{2}^{a} \bmod \mathscr{I} \Rightarrow\left(\kappa_{2}\right)_{a}^{m}=Q_{a b}^{m} \psi_{2}^{b}, & Q_{a b}^{m}=Q_{b a}^{m} .
\end{array}
$$

Case $s_{1}=s_{2}$. Assume we have chosen bases such that the entries of the first two columns of $\psi$ are all independent. Then it is clear that $\kappa_{2}=0$ since $\psi_{1}^{b}, \psi_{2}^{b}$ are all independent. Since no other new forms appear in the 
structure equations, there are no new classes of solutions. If $s_{2}=s_{1}-1$ then assume all forms in the first two columns are independent except for $\psi_{s_{1}}^{2}$. This implies $Q_{a \rho}^{m}=0,1 \leq \rho \leq s_{2}$, and, by the symmetry in $Q$, that only $Q_{s_{1} s_{1}}^{m}$ could be nonzero. Thus $\left(\kappa_{2}\right)_{\rho}^{m}=0 \Rightarrow P_{\rho b}^{m}=0$. The only possible nonzero entries of $\kappa_{2}$ are

$$
\left(\kappa_{2}\right)_{s_{1}}^{m}=Q_{s_{1} s_{1}}^{m} \psi_{2}^{s_{1}}=Q_{s_{1} s_{1}}^{m}\left(C_{a} \psi_{1}^{a}\right),
$$

where the $C_{a}$ are given constants (were $\psi_{2}^{s_{1}}$ to depend upon any $\psi_{2}^{\rho}$, we would already have $\kappa_{2}=0$ ). On the other hand, we must have

$$
\left(\kappa_{2}\right)_{s_{1}}^{m}=P_{s_{1} a}^{m} \psi_{1}^{a}=P_{s_{1} s_{1}}^{m} \psi_{1}^{s_{1}},
$$

where the second equality is because the other $P$ terms must be zero.

The above implies that to have nonzero $\kappa_{2}$ we must have $C_{\rho}=0$ but now by rechoosing bases we may assume $C_{s_{1}}=0$ which implies $\kappa_{2}=0$.

Remark. This is the best result of this type one can expect because adding vertical zeros to the $2 \times 2$ Cauchy-Riemann tableau does give new solutions. Thus adding zeros to an m-tableau that is a $2 \times 2$ CauchyRiemann tableau stacked below any m-tableau will also have new classes of solutions when zeros are added vertically.

Theorem 5.2. If an involutive $m$-tableau $A$ is equivalent to an $m$-tableau having two rows of independent entries or one having two rows, all of whose entries are independent but one, and $n>2$, then adding 'horizontal zeros' to $A$ will not enlarge its class of corresponding $m$-subsets. In particular, adding horizontal zeros to the Dirac or Dirac ${ }^{*}$ m-tableaux (except for C-type) and special Lagrangian m-tableaux does not enlarge the class of solution $m$-subsets.

Proof. Write $\psi_{0}=(\psi, \tilde{\psi})$, and $\tau=\left(\begin{array}{ll}\tau_{1} & \tau_{2} \\ \tau_{3} & \tau_{4}\end{array}\right)$. Say the system for $\mathrm{m}-$ subsets with $A$-type tableau is $\mathscr{I}=\left\{\theta^{r}\right\}$, with independence condition $\Omega$. Then the system for m-subsets with ( $A \quad 0)$-type tableau is $\mathscr{I}=$ $\left\{\theta^{r}, \tilde{\psi}\right\}$. Now proceed as with Theorem 5.1. q.e.d.

A related question to adding zeros is the following: Given $G \subset \mathrm{SO}(n+s)$ and $H \subset G$ such that $G / H \subset G_{n, n+s}$, and $G / H$ is an m-subset, do there exist other representations $\rho(G) \subset \mathrm{SO}(N)$ such that $\rho(G / H) \subset G_{M, N}$ is an m-subset? This occurs for $\mathrm{SU}(m)$ as shown in Theorem 3.10, but the above theorems suggest that it is a rare phenomenon.

Infinitesimally, if $G / H \subset G_{n, n+s}$ is an m-subset, write $\mathfrak{g}=\mathfrak{h} \oplus \mathfrak{m}$ such that

$$
\mathfrak{h}=\left(\begin{array}{cc}
\mathfrak{h}_{1} & 0 \\
0 & \mathfrak{h}_{2}
\end{array}\right)
$$


where $\mathfrak{h}_{1}, \mathfrak{h}_{2}$ are not necessarily independent, and

$$
\mathfrak{m}=\left(\begin{array}{cc}
Q_{1} & -{ }^{t} A \\
A & Q_{2}
\end{array}\right),
$$

where $A$ is an m-tableau and $Q_{1}, Q_{2}$ are linearly dependent on $A$ ( $Q_{j}=$ 0 if $G / H$ is symmetric). The question is: Does there exist a representation $\rho: G \rightarrow \mathrm{SO}(N)$ such that

$$
\rho(\mathfrak{m})=\left(\begin{array}{cccc}
* & * & -{ }^{t} A & 0 \\
* & * & 0 & 0 \\
A & 0 & * & * \\
0 & 0 & * & *
\end{array}\right) ?
$$

Finding an example of this for a non-Cauchy-Riemann m-tableau would also give the first example of a non-Cauchy-Riemann m-tableau having more than one type of solution m-subset. By Theorems 5.1 and 5.2 this cannot occur for the other faces studied in [8]. One may think of Theorems 5.1 and 5.2 as giving representation-theoretic information about $\mathrm{SU}(n)$, $G_{2}$, and $\operatorname{Spin}(7)$.

A representation-theoretic way of thinking of why Theorems 5.1 and 5.2 ought to be true in these cases is that in the relevant representations, all the weights of the representation interact with the m-tableau under the Lie bracket. (Compare with the codimension two representations of $\mathrm{SU}(n+1)$.

\section{Appendix: Calibrating the $\Sigma$-manifolds for $\Sigma$, a $\mu(\mathrm{SO}(3))$ orbit of a special codimension-two plane}

Let $\mu: \mathrm{SO}(3) \rightarrow \mathrm{SO}(2 n+1)$ be the unique irreducible representation. Under the action of $\mu(\mathrm{SO}(3))$, not all $(2 n-1)$-planes are the same, some have a circle in $\mathrm{SO}(3)$ whose action preserves the plane. Call these $(2 n-1)$-planes special. Let $\Sigma$ be the orbit in $G_{2 n-1,2 n+1}$ of a special $(2 n-1)$-plane. The associated $\Sigma$-manifolds are studied in detail in [9]. They are described in terms of holomorphic data on an auxiliary manifold $\mathscr{L}$, the space of special lines in $\mathbf{R}^{2 n+1}$ (compare with Theorem II.3.10). We have the following double fibration picture:

$$
\mathscr{L}^{\mathscr{F} \cong \mu(\mathrm{SO}(3)) \times \mathbf{R}^{2 n+1}} \searrow_{\mathbf{R}^{2 n+1},}
$$

where $\mathscr{F}$ is the $\mu(\mathrm{SO}(3))$ coframe bundle and $\mathscr{L} \cong \mathbf{C} \times \mathbf{C P} \mathbf{P}^{1}$ is a quotient space of $\mathscr{F}$ on which the differential system for lifts of $\Sigma$-manifolds to $\mathscr{F}$ 
are naturally defined. The $\Sigma$-manifolds $M$ are obtained by lifting certain complex curves in $\mathscr{L}$ to the coframe bundle $\mathscr{F}$, and projecting them to $\mathbf{R}^{2 n+1}$. Moreover, in [9], we give a local representation formula explicitly for the $n=2$ case, using coordinates adapted to the differential system for the $\Sigma$-manifolds. $\mathscr{F}$ is given coordinates $z, w, y \in \mathbf{C}, s, t \in \mathbf{R}$, where $z, w, y$ descend to also give coordinates on $\mathscr{L}$. In these coordinates, solutions are given by complex curves $w=h(z), y=h^{\prime}(z)$ which are then lifted to $\mathscr{F}$ and projected down to $\mathbf{R}^{5}$.

Theorem. Let $\Sigma$ be a $\mu(\mathrm{SO}(3))$ orbit of a special codimension-two plane. Then the associated $\Sigma$-manifolds are calibrated (or twisted calibrated) as submanifolds of $\mathbf{R}^{2 n+1}$ minus a small subset (where the meaning of 'small subset' will be made clear in the proof).

Proof. For the moment assume $2 n+1=5$. Following the notation of [9], let

$$
\left(\begin{array}{c}
\omega^{0} \\
\omega^{1} \\
\omega^{2} \\
\omega^{3} \\
\omega^{4}
\end{array}\right), \quad \frac{1}{2}\left(\begin{array}{cc}
i \rho & -\bar{\pi} \\
\pi & -i \rho
\end{array}\right)
$$

denote the tautological and connection forms on $\mathscr{F}$, respectively (where we are using the isomorphism $\mathfrak{s o}(3) \cong \mathfrak{s u}(2))$. Let $\eta=\omega^{1}+i \omega^{2}$ and $\theta=\omega^{3}+i \omega^{4}$. Let $\Upsilon^{7}$ denote the quotient manifold $\mathscr{F} / \mathrm{SO}(2)$. It has coordinates $z, w, y \in \mathbf{C}$ and $t \in \mathbf{R}$. Let $\Lambda_{h}^{5} \subset \Upsilon$ be the submanifold obtained by setting $w=h(z)$, where $h(z)$ is a given holomorphic function specifying a $\Sigma$-manifold $M$ as described above.

On $\Upsilon$ we have the well-defined quadratic form $Q=\left(\omega^{0}\right)^{2}+\cdots+\left(\omega^{4}\right)^{2}$ which descends from the pullback of the metric on $\mathbf{R}^{5}$ to $\mathscr{F}$.

Endow $\Lambda$ with the metric obtained by restricting $Q$ to $\Lambda$. This makes the map $\Lambda \stackrel{p}{\rightarrow} \mathbf{R}^{5}$ an isometry onto its image (where $p$ is the restriction of the projection $\Upsilon \rightarrow \mathbf{R}^{5}$ ).

Let $M$ be the $\Sigma$-manifold given by $w=h(z), y=h^{\prime}(z)$. Notice that the adapted lift of $M$ to $\Upsilon$ actually lies in $\Lambda$ (it is the submanifold of $\Lambda$ given by $\left.y=h^{\prime}(z)\right)$.

Consider the three-form

$$
\Omega:=\frac{i}{2} \omega^{0} \wedge(\eta \wedge \bar{\eta}+\theta \wedge \bar{\theta})
$$

which is well defined on $\Upsilon$. It has comass one because $\underline{\Omega}:=d x^{0} \wedge$ $\left(d x^{1} \wedge d x^{2}+d x^{3} \wedge d x^{4}\right)$ has comass one and at any point $\Omega$ is just $\underline{\Omega}$ acted on by an element of $\mu(\mathrm{SO}(3))$ (which preserves comass). 
Restricted to $\Lambda, \Omega$ has comass less than or equal to one, but in fact it has comass one because it is a volume form on $p^{-1}(M)$. Moreover, on $\Lambda, \Omega$ is closed as

$$
d \Omega=\frac{i \sqrt{3}}{4}(\bar{\pi} \wedge \eta+\pi \wedge \bar{\eta}) \wedge \theta \wedge \bar{\theta},
$$

but examining the coordinate expressions (4.1) and (4.4) in [9], we see $\pi$ is a function times $d z$, and when restricted to $\Lambda$, so is $\theta$. Thus $\left.\Omega\right|_{\Lambda}$ defines a calibration on $\Lambda$ which calibrates $p^{-1}(M)$ and since $p$ is an isometry, if it is injective, we may pull $\Omega$ back to $p(\Lambda)$ via $p^{-1}$ to get a nonconstant coefficient calibration on $p(\Lambda) \subseteq \mathbf{R}^{5}$ which calibrates $M$. Thus $M$ is minimizing among submanifolds of $p(\Lambda)$. If $p$ is not injective then $\Omega$ may instead define a twisted calibration on $p(\Lambda)$ (see [12]). In this case $M$ is minimizing among submanifolds of $p(\Lambda)$ having a lift to $\Lambda$. q.e.d.

The same proof as above works for any of the codimension two cases, because the coordinate expression for $\theta$ was independent of the dimension of the submanifold.

Remark. These calibrations are reminiscent of, but distinct from, those used by Murdoch [12] and Lawlor [10]. All are nonparallel, basically calibrate one submanifold, and are essentially defined on the normal bundle of the submanifold of interest and 'pushed down' to $\mathbf{R}^{n}$. Murdoch constructs a different local twisted calibration to calibrate the cone on the Veronese than the one here. As with the calibrations here, his is not defined everywhere, but he determined the exact set on which his calibration was not defined. Lawlor constructs nonparallel calibrations to calibrate cones, which are defined on all of $\mathbf{R}^{n}$, and is thus able to prove those cones are globally minimizing. He is able to extend his calibrations to all of $\mathbf{R}^{n}$ by having them vanish off a neighborhood of the cone. One might hope that his methods could be applied to the calibrations here. The nonparallel calibrations used here are the first used to calibrate anything other than cones or graphs of hypersurfaces.

\section{References}

[1] J. F. Adams, Vector fields on spheres, Ann. of Math. (2) 75 (1962) 603-632.

[2] R. L. Bryant, Conformal and minimal immersions of compact surfaces into the 4-sphere, J. Differential Geometry 17 (1982) 455-473.

[3] __ Some remarks on austere submanifolds, preprint.

[4] R. L. Bryant, S. Chern, R. Gardner, H. L. Goldschmidt \& P. Griffiths, Exterior differential systems, Springer, Berlin, 1991.

[5] R. L. Bryant \& R. Harvey, Submanifolds in hyper-Kähler geometry, J. Amer. Math. Soc. 1 (1989) 1-31. 
[6] R. O. Buchweitz, D. Eisenbud, \& J. Herzog, Cohen-Macaulay modules on quadrics, singularities, representations of algebras, and vector bundles, Lecture Notes in Math., Vol. 1273, Springer, Berlin, 1987, 58-116.

[7] H. Federer, Geometric measure theory, Springer, New York, 1969.

[8] R. Harvey \& H. B. Lawson, Calibrated geometries, Acta Math. 148 (1982) 47-157.

[9] J. M. Landsberg, Minimal submanifolds of $\mathbf{E}^{2 n+1}$ arising from degenerate $\mathrm{SO}(3)$ orbits on the Grassmannian, Trans. Amer. Math. Soc. 325 (1991) 101-118.

[10] G. Lawlor, A sufficient criterion for a cone to be area minimizing, Ph.D. thesis, Stanford University, 1988.

[11] M. Micallef, Stable minimal surfaces in Euclidean space, J. Differential Geometry 19 (1984) 57-84.

[12] T. Murdoch, Twisted calibrations and the cone over the veronese, Ph.D. thesis, Rice University, 1988.

[13] D. Nance, Sufficient conditions for a pair of $n$-planes to be area-minimizing, Math. Ann. 279 (1987) 161-164.

[14] R. Osserman, Survey of minimal surfaces, Van Nostrand, New York, 1969.

[15] J. Roe, Elliptic operators, topology and asymptotic methods, Pitman Research Notes in Math., Vol. 179, Longview Scientific and Technical, 1988.

University of Pennsylvania 
